\title{
Dietary components and risk of cardiovascular disease and all-cause mortality: A review of evidence from meta-analyses
}

Running title: Dietary components and risk of CVD and mortality

Chun Shing Kwok MBBS MSc BSc, ${ }^{1,2}$ Martha Gulati MD, ${ }^{3}$ Erin D Michos MD, ${ }^{4}$ Jessica Potts PhD,${ }^{1}$ Pensee Wu MD, ${ }^{1,2}$ Lorraine Watson, ${ }^{5}$ Yoon K Loke MD,${ }^{6}$ Christian Mallen BMedSci BMBS MMedSci MPhil PhD, ${ }^{5}$ Mamas A Mamas BMBCh DPhil ${ }^{1,2}$

1. Keele Cardiovascular Research Group, Research Institute for Primary Care \& Health Sciences, Keele University, Stoke-on-Trent, UK.

2. Royal Stoke University Hospital, Stoke-on-Trent, UK.

3. University of Arizona College of Medicine-Phoenix, Phoenix, Arizona, USA.

4. Department of Medicine/Cardiology, The Johns Hopkins Ciccarone Center for the Prevention of Heart Disease, Baltimore, Maryland, USA.

5. Research Institute for Primary Care \& Health Sciences, Keele University, Stoke-on-Trent, UK.

6. Norwich Medical School, University of East Anglia, UK.

\section{Corresponding author:}

Dr Chun Shing Kwok

Keele Cardiovascular Research Group

Centre for Prognosis Research, Institute of Primary Care and Health Sciences

David Weatherall Building, Keele University, Newcastle-under-Lyme ST5 5BG

Email: shingkwok@doctors.org.uk

Tel: +44 (0)1782 $671653 \quad$ Fax: +44 (0)1782 674467

Keyword: diet, epidemiology, systematic review

Word count: 5,000 


\begin{abstract}
Aims: The optimal diet for cardiovascular health is controversial. The aim of this review is to summarize the highest level of evidence and rank the risk associated with each individual component of diet within its food group.

Methods and results: A systematic search of PudMed was performed to identify the highest level of evidence available from systematic reviews or meta-analyses that evaluated different dietary components and their associated risk of all-cause mortality and cardiovascular disease (CVD). A total of 16 reviews were included for dietary food item and all-cause mortality and 17 reviews for CVD. Carbohydrates were associated with reduced risk of all-cause mortality (whole grain bread RR 0.85(95\%CI 0.82-0.89), breakfast cereal RR 0.88(0.83-0.92), oats/oatmeal RR 0.88(0.83-0.92)). Fish consumption was associated with a small benefit (RR 0.98 (0.97-1.00)) and processed meat appeared to be harmful (RR 1.25(1.07-1.45)). Root vegetables (RR 0.76(0.66-0.88)), green leafy vegetables/salad (RR 0.78(0.71-0.86)), cooked vegetables (RR 0.89(0.80-0.99)) and cruciferous vegetables (RR 0.90(0.85-0.95)) were associated with reductions in all-cause mortality. Increased mortality was associated with consumption of tinned fruit (RR 1.14(1.07-1.21)). Nuts were associated with a reduced risk of mortality in a dose response relationship (all nuts RR 0.78(0.72-0.84), tree nuts RR 0.82(0.75-0.90), and peanuts RR 0.77(0.69-0.86)). For CVD, similar associations for benefit were observed for carbohydrates, nuts and fish, but red meat and processed meat were associated with harm.
\end{abstract}

Conclusions: Many dietary components appear to be beneficial for CVD and mortality, including grains, fish, nuts and vegetables, but processed meat and tinned fruit appear to be harmful. 


\section{Introduction}

Cardiovascular disease (CVD) is a major global cause of health loss. ${ }^{1}$ Dietary habits, influence cardiovascular risk either through an effect of risk factors such as serum cholesterol, blood pressure, body weight and diabetes or through an effect independent of these risk factors. ${ }^{2}$ However, there is still controversy surrounding the optimal diet for cardiovascular health ${ }^{3}$ There has been exponential growth in the nutritional literature evaluating diet and cardiovascular disease. There have been reviews for specific food groups and their influence on cardiovascular health ${ }^{4}$ and further reviews of individual components of diet such as fish intake, ${ }^{5}$ cheese intake, ${ }^{6}$ butter $^{7}$ and less frequently consumed components such as soy products. ${ }^{8}$ One of the advantages of evaluating individual food components, is that overall diary patterns may mask the potential effects of individual food components. ${ }^{9}$ Nevertheless, as healthcare professionals it is necessary to give more holistic dietary advice rather than just focusing on individual food items / categories. There has yet to be a single review that has collated all available evidence from prior quality meta-analyses evaluating dietary components and risk of cardiovascular disease and all-cause mortality.

We conducted an up-to-date review of systematic reviews and meta-analyses on individual components of diet and their risk of cardiovascular disease and mortality. The aim of this review is to collectively summarize the highest level of evidence from previously conducted systematic reviews and meta-analyses and rank the risk associated with each individual component of diet within its food group.

\section{Methods}

\section{Search and study identification}

We carried out a review of the literature to identify the best evidence evaluating individual dietary components and risk of cardiovascular disease or mortality.

We began by identifying the broad categories of food after reviewing the "Eatwell Guide" in the United Kingdom, ${ }^{10}$ "The Five Food Groups" in the 2015-2020 Dietary Guidelines for Americans ${ }^{11}$ and the "Food Guide Pyramid" from the Center for Nutrition Policy and Promotion in the United States. ${ }^{12}$ Once the main groups of food were identified each individual component in a typical Western diet was determined and shown in Supplementary Table 1 .

For each individual component of diet, we searched for and identified the most recent and highest quality systematic review and meta-analysis evaluating the dietary component and its associated risk of adverse outcomes. This was a two-step process where first a search was performed and screened independently by two reviewers (CSK and either PW or JP). 
The search was performed on 13 August 2018 and we used each food category in Supplementary Table 1 as a key word on the Pubmed search. We chose to include the review with the most studies because the number of studies was part of our evidence grading criteria. The quality of the evidence for a systematic review of a food item was graded according to a modified criteria based on Grosso et al. ${ }^{13}$ The grading method has 4 levels where level 1 represents the highest level of evidence (convincing) and level 4 represents the lowest level of evidence (limited/contrasting). The exact method of grading the reviews based on inclusion of prospective cohorts, number of studies and the presence of statistical heterogeneity $\left(\mathrm{I}^{2} \leq 30 \%\right.$ vs $\left.\mathrm{I}^{2}>30 \%\right)$ is shown in Supplementary Table 2.

Included studies had to have the dietary component of interest and some form of quantitative association with either cardiovascular disease or mortality. Food item consumption and its association with outcome can be quantified as dose-response relationship and highest compared to lowest consumers of food items. We chose studies that considered a dose-response relationship where available.

The search process as described in this paragraph was conducted in August 2018. We initially searched PubMed using the Clinical Queries option to identify systematic reviews using the dietary component as the search term along with the terms related to outcomes. These outcome terms are: (death OR mortality OR stroke OR cerebrovascular disease OR cerebrovascular accident OR coronary heart disease OR ischemic heart disease OR ischaemic heart disease OR coronary artery disease OR acute myocardial infarction OR acute coronary syndrome OR heart failure OR cardiac failure OR cardiac insufficiency). The results of the search process are shown in Supplementary Table 1.

\section{Evidence synthesis}

Statistical analysis was performed by presenting all the results and ranking them according to effect within each food group. For each included meta-analysis or review for the specific foods groups, we extracted the Relative Risks (RR) and 95\% confidence intervals (95\% CI) from the most adjusted models presented in the review; the evidence of heterogeneity $\left(\mathrm{I}^{2}\right)$ was obtained from the original source meta-analyses and reported in our Table 1. We also collected information on the quality assessments of the reviews. Results are presented numerically in Tables and Graphically in Figures. For graphical representation, the studies which reported associations of increased risk of harm were colored in red, those which showed beneficial associations were colored in green, and those which showed no statistical difference were colored in yellow. We performed additional analysis considering the impact of sex-specific differences in outcomes. 


\section{Results}

A total of 3,011 studies were reviewed from the search shown in Supplementary Table 1. After detailed review of relevant studies, a total of 16 reviews ${ }^{7,14-28}$ were included for all-cause mortality and 17 reviews , $^{7,14,17-20,22,24-32}$ for cardiovascular disease (Supplementary Figure 1).

Supplementary Table 3 shows the quality assessment conducted in each included review. The grading of the evidence based on the criteria in Supplementary Table 3 suggested that many analyses showed the lowest or most limited (level 4) evidence mainly because there were fewer than 4 studies (Supplementary Table 4). However, for all-cause mortality, level 2 evidence was present for refined grains, green leafy vegetables/salad and tinned fruit. For cardiovascular disease, there was only level 2 evidence for fish. None of the metaanalyses were based on randomized controlled trial data.

Table 1 and Figure 1 show the food items within different food groups and their risk of all-cause mortality. For carbohydrates, there were 2 or fewer studies for the assessment of whole grain bread, pasta, whole grain breakfast cereals, oats/oatmeal. In the dose-response analysis all of these food items were associated with reduced risk of all-cause mortality (whole grain bread RR 0.85 (95\% CI 0.82-0.89), pasta RR 0.85 (0.74-0.99), whole grain breakfast cereal RR 0.88 (0.83-0.92), oats/oatmeal RR 0.88 (0.83-0.92). Both intake of refined grains and fibre were associated with a significant dose response reduction in allcause mortality (RR 0.95 (0.91-0.99), 4 studies and RR 0.90 (0.86-0.94), 8 studies, respectively). Rice was evaluated in 5 studies in the highest consumer compared to the lowest consumer analysis and no significant difference in mortality was observed.

Among meat, eggs and fish, fish consumption was associated with a small benefit for mortality (RR 0.98 (0.97-1.00)) and processed meat appeared to be harmful (RR 1.25 (1.071.45)). No significant differences were observed for white meat, red meat and eggs. Among fruits and vegetables, root vegetables (RR 0.76 (0.66-0.88), 1 study), green leafy vegetables/salad (RR 0.78 (0.71-0.86), 7 studies), cooked vegetables (RR 0.89 (0.80-0.99), 4 studies) and cruciferous vegetables (RR 0.90 (0.85-0.95), 6 studies) were associated with reductions in all-cause mortality. There was an association for increased mortality with a dose-response consumption of tinned fruit (RR 1.14 (1.07-1.21), 4 studies). Comparing the highest and lowest consumers of alcohol there appeared to be reduction in all-cause mortality among the highest consumers (RR 0.87 (0.83-0.92), 31 studies). Coffee also showed a doseresponse association for reduced risk of all-cause mortality (RR 0.96 (0.94-0.97), 16 studies). For dairy products, there was no significant difference in risk of mortality with yogurt, 
cheese, milk or butter consumption. The data from nuts appeared to be associated with reduced risk of mortality in a dose response relationship (all nuts RR 0.78 (0.72-0.84), 16 studies, tree nuts RR 0.82 (0.75-0.90), 4 studies and peanuts RR 0.77 (0.69-0.86), 5 studies).

The associations between cardiovascular disease and food items are shown in Figure 2 and Table 2. Among carbohydrates, there was a dose-response association for benefit for whole grain bread (RR 0.87 (0.80-0.95), 3 studies), whole grain breakfast cereals (RR 0.84 (0.78-0.90), 2 studies), bran (RR 0.85 (0.79-0.90, 2 studies) and fibre (RR 0.91 (0.88-0.94), 10 studies). Red meat (RR 1.15 (1.05-1.26), 6 studies) and processed meat (RR 1.24 (1.091.40), 6 studies) appeared to be harmful. Out of all the fruits and vegetables only 1 study on raw vegetables suggested a dose-response association of benefit (RR 0.86 (0.81-0.90)). Alcohol consumption for the highest compared to the lowest consumers showed an association of reduced risk of cardiovascular disease (RR 0.75 (0.70-0.80), 21 studies). Black tea was associated with a dose-response benefit for cardiovascular mortality (RR 0.92 (0.850.99), 7 studies). Dairy products (yogurt, cheese, milk and butter) showed no evidence of a dose response association for benefit or harm. Intake of nuts were associated with reduced risk of cardiovascular disease (all nuts RR 0.79 (0.70-0.88), 12 studies, tree nuts RR 0.75 (0.67-0.84), 3 studies, peanuts RR 0.64 (0.50-0.81), 5 studies). In addition, olive oil showed a dose-response benefit in cardiovascular disease RR 0.82 (0.70-0.96), 9 studies and soy products as compared by highest and lowest consumers showed lower risk of cardiovascular disease (RR 0.83 (0.75-0.93)). Finally, an association for a dose response benefit was observed for chocolate (RR 0.982 (0.972-0.992), 12 studies).

The additional analysis considering differences in results based on sex showed no major differences between men and women in most studies (Supplementary Table 5). 


\section{Discussion}

To facilitate clinician-patient communications regarding the impact of diet for cardiovascular health, we have summarized current evidence from the highest quality systematic reviews available by various food groups. We have shown that food components within food groups are associated with different risks for cardiovascular disease and all-cause mortality. Many fruits and vegetables which are presumed to be beneficial as a group actually lack strong evidence of cardiovascular benefit. The best evidence appears to support the intake of green leafy vegetables/salad to reduce all-cause mortality. On the other hand, processed meat appears to be harmful for both all-cause mortality and cardiovascular disease.

Our results are important as diet is complex and it appears that there may be dissonance between foods which are for beneficial for all-cause mortality and cardiovascular disease. We speculate that this may be because the major causes of all-cause mortality are likely a composite of cardiovascular disease and those of cancer etiology. While oxidative stress plays an important role in both atherosclerosis ${ }^{33}$ and oncogenesis ${ }^{34}$ and both cardiovascular disease and cancer share risk factors such as obesity, ${ }^{35}$ physical inactivity, diabetes $^{36}$ and smoking. ${ }^{37}$ Hypertension is common and strongly associated with cardiovascular disease but the evidence of its link to cancer is less strong. Dietary elements which affect blood pressure may have greater benefits for cardiovascular disease risk whilst food items that protect from oxidative stress may have a greater protective effect for cancer.

The consideration of individual foods and food components has been highlighted as a key approach use by the public when interpreting healthy eating messages. ${ }^{38} \mathrm{We}$ found that dietary nuts appear to be beneficial for both all-cause mortality and cardiovascular disease. Tree nuts and peanuts are foods rich in high-quality vegetable protein, fiber, minerals, tocopherols, phytosterols and phenoic compounds which beneficially impact health outcomes. ${ }^{39}$ Consumption of nuts are associated with a favorable fatty acid profile which is high in unsaturated fatty acids and low in saturated fatty acids which contributes to cholesterol lowering. ${ }^{40}$ Also, nuts have a tendency to lower body weight and fat mass and in the context of calorie-restricted diets, adding nuts promotes weight loss in obese subjects and improves insulin sensitivity. ${ }^{41}$ It has been further suggested that the benefits of the Mediterranean diet may be partly attributed to nuts. ${ }^{42}$ We believe more studies are need to examine different types of tree nuts as there was insufficient data on important nuts like almonds, cashews, macadamia nuts, pistachios and walnuts.

We found evidence that processed meat and tinned fruit may be harmful. The biggest difference among constituents of processed and unprocessed meat are sodium and nitrate 
which are $400 \%$ and $50 \%$ more per gram of meat. ${ }^{43}$ Blood pressure and peripheral vascular resistance increase with dietary sodium, and dietary sodium may also impair arterial compliance. ${ }^{44}$ It is further suggested that nitrates and their by-products may promote endothelial dysfunction, atherosclerosis and insulin resistance. ${ }^{45-47}$ For tinned fruit, it has been suggested that the population consuming tinned fruit tended to be male, older, report lower education level, have higher body mass index and more likely to have diabetes. ${ }^{48}$ Compared to fresh fruit, tinned fruit has added sugar which may contribute to cardiovascular mortality. ${ }^{49}$ There may also be concerns about bisphenol A which is greater in tinned fruit and the acidity of food cans may dissolve lead solder from food cans. ${ }^{48}$

There are inherent challenges and limitations in analyzing nutritional data from observational studies, yet such research has played a vital role over the years in identifying new links between food and health. ${ }^{50}$ First, it is possible that some of the food items assessed showed a non-linear dose-response relationship and estimates at high or very low doses may not be accurate. Second, multiple repeat measures are required to explore effects of variation on exposure over time so caution may be needed when interpreting risk of exposures measured only once at baseline. ${ }^{51}$ This may apply for items which are not consumed on a regular basis or food items where there is major variability such as a person who drinks alcohol regularly at low quantities daily versus a person who drinks less frequently but heavily. Third, some of the food items which show no association of benefit or harm may actually have an impact for the individual cardiovascular risk factors such as blood pressure or cholesterol levels and may be beneficial or harmful for some subgroups of the populations such as patients with diabetes. Fourth, while our results showed that certain foods appear to be beneficial or harmful it is important that these results should be taken in consideration of patients' overall nutrition status. Fifth, even though lifestyle and socioeconomic factors may be adjusted for in the cohort studies included in our review, it is likely there is residual confounding by sociodemographic and lifestyle factors. Patients who eat "healthier" foods are also more likely to be educated, have greater income, more likely to exercise regularly, more likely to be of normal weight and body mass index, more likely be a non-smoker and have better access to healthcare, and the collective effects of these factors may not be completely accounted for in the adjustments. Sixth, another important consideration is that the comparison group is not the same across each analysis. An obvious difficulty is that eating food is essential to health and wellbeing so it would not be possible to conduct a study comparing individual food items to consuming nothing and there is no obvious single food reference to compare to. Furthermore, there are other limitations such as self-reporting bias, 
recall bias, and heterogeneity in the way food intake was estimated among the studies. While dietary studies tend to disproportionately attract media attention and often the communicated result is that a specific food will cause or prevent a certain disease, the conclusions and results need to be scrutinized as the case of the current review and methodological limitations of these dietary studies make interpretations of a 'perfect food' very unlikely.

While the current study demonstrates that dietary components have different associations with adverse outcomes, it is important to recognize that our current study only considers the dietary component of associations with overall cardiovascular disease. There has been a study to suggest that the Mediterranean diet and adopting an active lifestyle show a synergistic effect in their inverse association with cardiovascular disease risk. ${ }^{52}$ Considering this finding, the overall cardiovascular disease risk likely incorporates a variety of factors which would contribute but may or may not further interact to modify the overall risk.

Our study has several limitations. While we were able to cover many different vegetables there was insufficient evidence for many meat types and nuts and there was no data on seafood other than fish. More importantly many reviews only had level 4 or limited evidence because there were fewer than 4 studies. Nevertheless, our review is important as it summarizes in a concise way the evidence for food items that are associated with all-cause mortality and cardiovascular disease. A further limitation is that we are unable to assess on the individual study level the impact of daily calorific content of foods and any clustering effects in dietary intake.

In conclusion, many food items appear to be beneficial in diet including nuts, whole grain foods and fiber. Within the fruit and vegetables category many foods presumed to be beneficial actually have insufficient evidence to suggest benefit in cardiovascular disease but there is modest evidence for benefit for raw vegetables, root vegetables, green leafy vegetables, cooked vegetables and cruciferous vegetables and all-cause mortality. Foods that appear harmful include processed meat and tinned fruit for all-cause mortality and processed meat and red meat for cardiovascular disease. Our review provides a comprehensive summary of the evidence of benefit or harm of food items which may help physicians better counsel their patients about dietary advice.

\section{Acknowledgement: None.}

\section{Funding: None.}




\section{Conflicts of interest: None.}

Authors' Contribution: CSK designed the study, concept and performed the data analysis. CSK, JP and PW were involved in the data collection. CSK wrote the first draft of the manuscript. All authors critically revised the manuscript and gave final approval and agree to be accountable for all aspects of work ensuring integrity and accuracy.

\section{References}

1. Roth GA, Johnson C, Abajobir A, et al. Global, regional and national burden of cardiovascular disease for 10 causes, 1990 to 2015. J Am Coll Cardiol 2017;70:1-25.

2. Verschuren WMM. Diet and cardiovascular disease. Curr Cardiol Rep 2012;14:701708.

3. Anand SS, Hawkes C, de Souza RJ, et al. Food comsumption and its impact on cardiovascular disease: importance of solutions focused on the globalized food system. J Am Coll Cardiol 2015;66:1590-1614.

4. Bechthold A, Boeing H, Schwedhelm C, et al. Food groups and risk of coronary heart disease, stroke and heart failure: A systematic review and dose-response metaanalysis of prospective studies. Crit Rev Food Sci Nutr. 2017;1-20.

5. Mozaffarian D, Rimm EB. Fish intake, contaminants, and human health: evaluating the risks and benefits. JAMA 2006;296:1885-99.

6. Chen GC, Wang Y, Tong X, et al. Cheese consumption and risk of cardiovascular disease: a meta-analysis of prospective studies. Eur J Nutr 2017;56:2565-2575.

7. Pimpin L, Wu JH, Haskelberg $\mathrm{H}$, et al. Is butter back? A systematic review and metaanalysis of butter consumption and risk of cardiovascular disease, diabetes and total mortality. PLoS One 2016;11:e0158118.

8. Yan Z, Zhang X, Li C, Jiao S, Dong W. Association between consumption of soy and risk of cardiovascular disease: A meta-analysis of observational studies. Eur J Prev Cardiol 2017;24:735-747.

9. Schulze Matthias B, Martínez-González Miguel A, Fung Teresa T, et al. Food based dietary patterns and chronic disease prevention BMJ 2018; 361:k2396.

10. GOV.UK. The Eatwell Guide. Available at: https://www.gov.uk/government/publications/the-eatwell-guide. Last accessed October 22, 2018.

11. Office of Disease Prevention and Health Promotion. 2015-2020 Dietary guidelines for Americans. Available at: https://health.gov/dietaryguidelines/2015/. Last accessed October 22, 2018.

12. United States Department of Agriculture Center for Nutrition Policy and Promotion. Food Guide Pyramid. Available at: https://www.cnpp.usda.gov/FGP. Last accessed October 22, 2018.

13. Grosso G, Godos J, Alvano F, Giovannucci EL. Coffee, caffeine, and health outcome: an umbrella review. Ann Rev Nutr 2017;37:131-156.

14. Aune D, Keum N, Gionvannucci E, et al. Whole grain consumption and risk of cardiovascular disease, cancer and all cause and cause specific mortality: systematic review and dose-response meta-analysis of prospective studies. BMJ 2016;353:i2716. 
15. Saneei P, Larijani B, Esmaillzadah A. Rice consumption, incidence of chronic diseases and risk of mortality: meta-analysis of cohort studies. Pub Health Nutr 2017;20:233-244.

16. Yang Y, Zhao LG, Wu Q, Ma X, Xiang XB. Association between dietary fiber and lower risk of all-cause mortality: A meta-analysis of cohort studies. Am J Epidemiol 2015; 181:83-91.

17. Jayedi A, Shab-Bidar S, Eimeri S, Djafarian K. Fish consumption and risk of allcause and cardiovascular mortality: a dose-response meta-analysis of prospective observational studies. Public Health Nutr 2018;21:1297-1306.

18. Abete I, Romaguera D, Vieira AR, de Munain AL, Norat T. Association between total, processed, red and white meat consumption and all-cause, CVD and IHD mortality: a meta-analysis of cohort studies. Br J Nutr 2014;112:762-775.

19. Xu L, Lam TH, Jiang CQ, et al. Egg consumption and the risk of cardiovascular disease and all-cause mortality: Guangzhou Biobank Cohort Study and meta-analysis. Eur J Nutr 2018. doi: 10.1007/s00394-018-1692-3.

20. Aune D, Giovannucci E, Boffetta P, et al. Fruit and vegetable intake and the risk of cardiovascular disease, total cancer and all-cause mortality-a systematic review and dose-response meta-analysis of prospective studies. Int J Epidemiol 2017;1029-1056.

21. Schwingshackl L, Schwedhelm C, Hoffmann G, Boeing H. Potatoes and risk of chronic disease: a systematic review and dose-response meta-analysis. Eur J Nutr 2018. doi: 10.1007/s00394-018-1774-2.

22. Ronksley PE, Brien SE, Turner BJ, et al. Association of alcohol consumption with selected cardiovascular disease outcomes: a systematic review and meta-analysis. BMJ 2011;342:d671.

23. Je Y, Giovannucci E. Coffee consumption and total mortality: a meta-analysis of twenty prospective cohort studies. Br J Nutr 2014;111:1162-1173.

24. Tang J, Zheng JS, Fang L, Jin Y, Cai W, Li D. Tea consumption and mortality of all cancers, CVD and all causes: a meta-analysis of eighteen prospective cohort studies. Br J Nutr 2015;114:673.

25. Narain A, Kwok CS, Mamas MA. Soft drinks and sweetened beverages and the risk of cardiovascular disease and mortality: a systematic review and meta-analysis. Int $\mathbf{J}$ Clin Pract 2016;70:791-805.

26. Guo J, Astrup A, Lovegrove JA, et al. Milk and dairy consumption and risk of cardiovascular diseases and all-cause mortality: dose-response meta-analysis of prospective cohort studies. Eur J Epidemiol 2017:32:269-287.

27. Aune D, Keum N, Giovannucci E, et al. Nut consumption and risk of cardiovascular disease, total cancer, all-cause and cause-specific mortality: a systematic review and dose-response meta-analysis of prospective studies. BMC Med 2016;14:207.

28. Alburto NJ, Ziolkovska A, Hooper L, et al. Effect of lower sodium intake on health: systematic review and meta-analyses. BMJ 2013;346:f1326.

29. Treapleton DE, Greenwood DC, Evans CE, et al. Dietary fibre intake and risk of cardiovascular disease: systematic review and meta-analysis. BMJ 2013;347:f6879.

30. Malerba S, Turati F, Galeone C. A meta-analysis of prospective studies and coffee consumption and mortality for all causes, cancers and cardiovascular diseases. Eur J Epidemiol 2013;28:527-539.

31. Martinez-Gonzalez MA, Dominguez LJ, Delgado-Rodriguez M. Olive oil consumption and risk of CHD and/or stroke: a meta-analysis of case-control, cohort and interventional studies. Br J Nutr 2014;112:248-259. 
32. Ren Y, Liu Y, Sung XZ, et al. Chocolate consumption and risk of cardiovascular disease: a meta-analysis of prospective studies. Heart 2018; doi: 10.1136/heartjnl2018-313131.

33. Kattoor AJ, Pothineni NVK, Palagiri D, Mehta JL. Oxidative stress in atherosclerosis. Curr Atheroscler Rep 2017;19:42.

34. Reuter S, Gupta SC, Mhaturvedi MM, Aggarwal BB. Oxidative stress, inflammation, and cancer: How are they linked? Free Radic Biol Med 2010;49:1603-1616.

35. Basen-Engquist K, Chang M. Obesity and cancer risk: recent review and evidence. Curr Oncol Rep 2011;13:71-76.

36. Vigneri P, Fasca F, Sciacca L, Pandini G, Vigneri R. Diabetes and cancer. EndocrineRelated Cancer 2009;16:1103-1123.

37. Carbone D. Smoking and cancer. Am J Med 1992;93:S13-17.

38. Bisogni CA, Jastran M, Seligson M, Thompson A. How people interpret healthy eating: contributions of qualitative research. J Nutr Educ Behav 2012;44:282-301.

39. Ros E. Health benefits of nut consumption. Nutrients 2010;2:652-682.

40. Kris-Etherton PM, Zhao G, Binkoski AE, Coval SM, Etherton TD. The effect of nuts on coronary heart disease risk. Nutrition Reviews 2001;59:103-111.

41. Rajaram S, Sabete J. Nuts, body weight and insulin resistance. Br J Nutr 2006;96:S79-S86.

42. Ros E. The Mediterranean Diet - Chapter 17 - Contribution of Nuts to the Mediterranean Diet. 2015;175-184.

43. Micha R, Michas G, Mozaffarian D. Unprocessed red and processed meats and risk of coronary artery disease and type 2 diabetes - an updated review of the evidence. Curr Atheroscler Rep 2012;14:515-524.

44. Sacks FM, Campos H. Dietary therapy in hypertension. N Engl J Med. 2010;362:2102-12.

45. Forstermann U. Oxidative stress in vascular disease: causes, defense mechanisms and potential therapies. Nat Clin Pract Cardiovasc Med. 2008;5:338-349

46. McGrowder D, Ragoobirsingh D, Dasgupta T. Effects of S-nitrosoN-acetylpenicillamine administration on glucose tolerance and plasma levels of insulin and glucagon in the dog. Nitric Oxide. 2001;5:402-412.

47. Portha B, Giroix MH, Cros JC, Picon L. Diabetogenic effect of Nnitrosomethylurea and N-nitrosomethylurethane in the adult rat. Ann Nutr Aliment. 1980;34:1143-51.

48. Aasheim ET, Sharp JS, Appleby PN, et al. Tinned fruit consumption and mortality in three prospective cohorts. PLoS One 2015;10:e0117796.

49. Yang Q, Zhang Z, Gregg EW, et al. Added Sugar Intake and Cardiovascular Diseases Mortality Among US Adults. JAMA Intern Med 2014;30341:1-9.

50. Mozaffarian S, Foroughi N. Dietary guidelines and health- is nutrition science up to the task? BMJ 2018; 360:k822 doi:10.1136/bmj.k822

51. Britton A, Marmot MG, Shipley MJ. How does variability in alcohol consumption over time affect the relationship with mortality and coronary heart disease? Addiction 2010;105:639-645.

52. Alvarez-Alvarez I, de Rojas JP, Fernandez-Montero A, Zazpe I, Ruiz-Canela M, Hidalgo-Santamaria M, Bes-Rastrollo M, Martinez-Gonzalez MA. Strong inverse associations of Mediterranean diet, physical activity and their combination with cardiovascular disease: The Seguimiento Universidad de Navarra (SUN) cohort. Eur J Prev Cardiol 2018;25:1186-1197. 


\section{Figure Legends}

Figure 1. Food items and risk of all-cause mortality

Figure 2. Food items and risk of cardiovascular disease

Table 1. Studies that evaluate food items and non-consumption of food items and all-cause mortality

Table 2. Studies that evaluate food items and non-consumption of food items and cardiovascular disease

\section{Appendices}

Supplementary Figure 1. Study selection process

Supplementary Table 1. Food categories, food components and search results

Supplementary Table 2. Grading of meta-analyses based on Grosso et al.

Supplementary Table 3: Quality assessments in the included systematic reviews and metaanalyses

Supplementary Table 4. Grading the quality of the evidence for each food component

Supplementary Table 5: Consideration of sex differences among included studies 
Figure 1. Food items and risk of all-cause mortality

\section{Food items and risk of all-cause mortality}

\begin{tabular}{lllll} 
Whole grain bread (per 90g/day) & RR $0.85(0.82-0.89)$ & & \\
Pasta (per 150g/day) & RR $0.85(0.74-0.99)$ & Fish or fish oil & RR $0.98(0.97-1.00)$ \\
Whole grain breakfast cereals (per 30g/day) & RR $0.87(0.84-0.90)$ & White meat (per 100g increase) & RR $0.90(0.73-1.11)$ \\
Oats/oatmeal (per 20g/day) & RR $0.88(0.83-0.92)$ & Red meat (per 100g) & RR $1.04(0.92-1.17)$ \\
Fibre (per 10g/day) & RR 0.90 (0.86-0.94) & Eggs (high vs low) & HR 1.09 (0.997-1.20) \\
Refined grains (per 90g/day) & RR 0.95 (0.91-0.99) & Processed meat (per 50g) & RR 1.25 (1.07-1.45) \\
\hline Rice (high vs low) & RR 0.97 (0.88-1.06) & &
\end{tabular}

Root vegetables (per 100g/day)

Gren

Cooked vegetables (per $100 \mathrm{~g} / \mathrm{day}$ )

Conciferous vegetables (per $100 \mathrm{~g} / \mathrm{day})$

Cruciferous vegetables (per $100 \mathrm{~g} / \mathrm{day}$ )

Raw vegetables (per $100 \mathrm{~g} / \mathrm{day}$ )

Mushrooms (per 100g/day) RR $0.91(0.81-1.02)$

(per 100g/day) RR $0.74(0.46-1.20)$

RR $0.76(0.40-1.46)$

RR 0.80 (0.64-1.01)

Citrus (per $150 \mathrm{~g} /$ day)

(Per $100 \mathrm{~g} / \mathrm{day}) \quad$ RR $0.95(0.89-1.02)$

Non-cruciferous vegetables (per 100g/day)

Bananas (per 100g/day)

RR $0.95(0.80-1.14)$

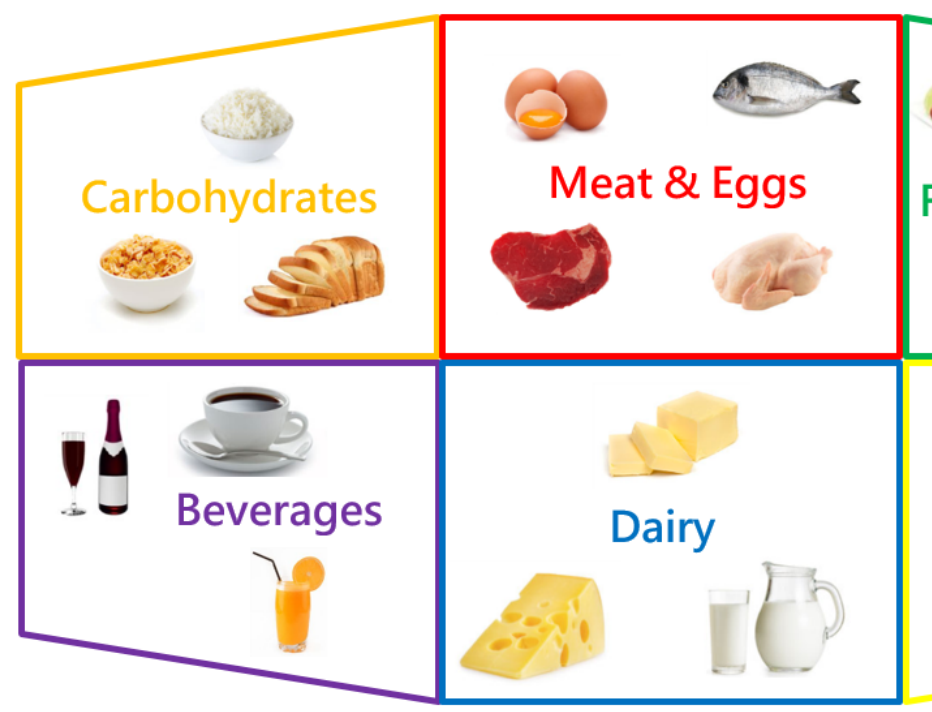

$\begin{array}{llll}\text { Alcohol (high vs low) } & \text { RR 0.87 (0.83-0.92) } & & \\ \text { Fruit juice (per 100g/day) } & \text { RR 0.88 (0.84-0.92) } & \text { Yogurt (per 50g/day) } & \text { RR 0.97 (0.85-1.11) } \\ \text { Coffee (per cup) } & \text { RR 0.96 (0.94-0.97) } & \text { Cheese (per 10 g/day) } & \text { RR 0.99 (0.96-1.01) } \\ \text { Green tea (per cup) } & \text { RR 0.96 (0.94-0.98) } & \text { Milk (per 244g/day) } & \text { RR } 1.00(0.93-1.07) \\ \text { Black tea (per cup) } & \text { RR 0.97 (0.94-0.99) } & \text { Butter (per 14g/day) } & \text { RR } 1.01(1.00-1.03)\end{array}$

Artificially sweetened beverages (high vs low) RR $109(0.92-1.30)$

Fruits \& Vegetables

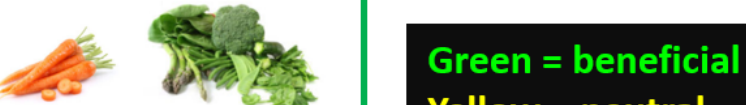

Yellow $=$ neutral

Red = harmful

Nuts \& other

$\begin{array}{ll}\text { Nuts (per 28g/day) } & \text { RR } 0.78(0.72-0.84) \\ \text { Tree nuts (per 10g/day) } & \text { RR } 0.82(0.75-0.90) \\ \text { Peanut (per 10g/day) } & \text { RR } 0.77(0.69-0.86) \\ \text { Peanut butter (per 10g/day) } & \text { RR } 0.94(0.86-1.02) \\ \text { Salt (high vs low) } & \text { RR } 1.06(0.94-1.20)\end{array}$


Figure 2. Food items and risk of cardiovascular disease 


\section{Food items and risk of cardiovascular disease}

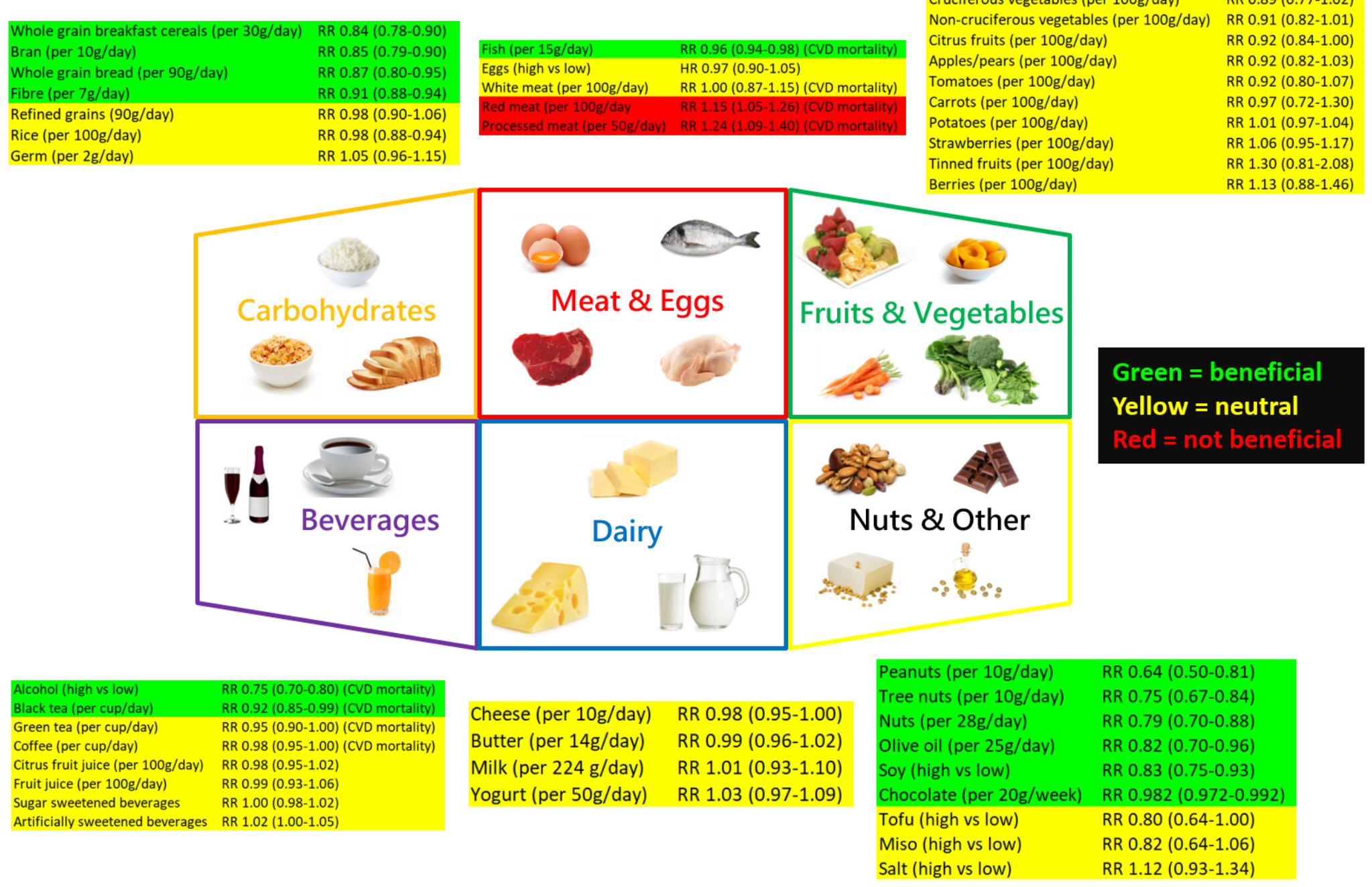


Table 1: Studies that evaluate food items and non-consumption of food items and all-cause mortality

\begin{tabular}{|c|c|c|c|c|c|c|}
\hline Food group & Food item & $\begin{array}{l}\text { Number } \\
\text { of } \\
\text { studies }\end{array}$ & $\begin{array}{l}\text { Sample } \\
\text { size }\end{array}$ & Inclusion criteria & $\begin{array}{l}\text { Risk estimate and statistical } \\
\text { heterogeneity. }\end{array}$ & Reference \\
\hline \multirow[t]{7}{*}{ Carbohydrate } & Whole grain bread & 2 & 153,858 & $\begin{array}{l}\text { Prospective cohort } \\
\text { studies up to Apr } 2016\end{array}$ & $\begin{array}{l}\text { Dose-response per } 90 \mathrm{~g} / \mathrm{day} \\
\text { RR } 0.85(0.82-0.89), \mathrm{I}^{2}=0 \%\end{array}$ & \multirow[t]{5}{*}{ Aune $2016^{14}$} \\
\hline & Pasta & 2 & 265,457 & $\begin{array}{l}\text { Prospective cohort } \\
\text { studies up to Apr } 2016\end{array}$ & $\begin{array}{l}\text { Dose-response per } 150 \mathrm{~h} / \text { day } \\
\text { RR } 0.85(0.74-0.99), \\
\mathrm{I}^{2}=54 \% .\end{array}$ & \\
\hline & $\begin{array}{l}\text { Whole grain breakfast } \\
\text { cereal }\end{array}$ & 2 & 206,200 & $\begin{array}{l}\text { Prospective cohort } \\
\text { studies up to Apr } 2016\end{array}$ & $\begin{array}{l}\text { Dose-response per } 30 \mathrm{~g} / \mathrm{day} \\
\text { RR } 0.87(0.84-0.90), \mathrm{I}^{2}=0 \% .\end{array}$ & \\
\hline & Oats/oatmeal & 1 & 120,010 & $\begin{array}{l}\text { Prospective cohort } \\
\text { studies up to Apr } 2016\end{array}$ & $\begin{array}{l}\text { Dose-response per } 20 \mathrm{~g} / \text { day } \\
\text { RR } 0.88(0.83-0.92) \text {. }\end{array}$ & \\
\hline & Refined grain & 4 & 163,634 & $\begin{array}{l}\text { Prospective cohort } \\
\text { studies up to Apr } 2016\end{array}$ & $\begin{array}{l}\text { Dose-response per } 90 \mathrm{~g} / \text { day } \\
\text { RR } 0.95(0.91-0.99), \\
I^{2}=20 \% \text {. }\end{array}$ & \\
\hline & Rice & 5 & 453,723 & $\begin{array}{l}\text { Cohort studies up to July } \\
2014\end{array}$ & $\begin{array}{l}\text { High vs low intake RR } 0.97 \\
(0.88-1.06), \mathrm{I}^{2}=39.4 \% .\end{array}$ & Saneei $2017^{15}$ \\
\hline & Fibre & 8 & 875,390 & $\begin{array}{l}\text { Prospective cohort } \\
\text { studies up to May } 2014 .\end{array}$ & $\begin{array}{l}\text { Dose-response per } 10 \mathrm{~g} / \text { day } \\
\text { RR } 0.90(0.86-0.94) \\
I^{2}=77.2 \%\end{array}$ & Yang $2015^{16}$ \\
\hline \multirow[t]{4}{*}{ Meat \& eggs } & Fish & 14 & 911,348 & $\begin{array}{l}\text { Prospective cohort } \\
\text { studies up to Sept } 2016\end{array}$ & $\begin{array}{l}\text { Dose-response per } 20 \mathrm{~g} / \text { day } \\
\text { RR } 0.98(0.97-1.00), \\
\mathrm{I}^{2}=81.9 \% \text {. }\end{array}$ & Jayedi $2018^{17}$ \\
\hline & White meat & 5 & $1,156,644$ & $\begin{array}{l}\text { Prospective cohort } \\
\text { studies up to Aug } 2013\end{array}$ & $\begin{array}{l}\text { Dose-response per } 100 \mathrm{~g} / \text { day } \\
\text { RR } 0.90(0.73-1.11), \\
I^{2}=92.1 \% \text {. }\end{array}$ & \multirow[t]{3}{*}{ Abete $2014^{18}$} \\
\hline & Red meat & 6 & 1,277986 & $\begin{array}{l}\text { Prospective cohort } \\
\text { studies up to Aug } 2013\end{array}$ & $\begin{array}{l}\text { Dose-response per } 100 \mathrm{~g} / \text { day } \\
\text { RR } 1.04(0.92-1.17), \mathrm{I}^{2}=95 \% \text {. }\end{array}$ & \\
\hline & Processed meat & 5 & $1,143,696$ & $\begin{array}{l}\text { Prospective cohort } \\
\text { studies up to Aug } 2013\end{array}$ & $\begin{array}{l}\text { Dose-response per } 50 \mathrm{~g} / \text { day } \\
\text { RR } 1.25(1.07-1.45) \text {, }\end{array}$ & \\
\hline
\end{tabular}




\begin{tabular}{|c|c|c|c|c|c|c|}
\hline & & & & & $\mathrm{I}^{2}=95.7 \%$ & \\
\hline & Eggs & 4 & 853,974 & $\begin{array}{l}\text { Prospective cohort } \\
\text { studies up to Mar } 2016\end{array}$ & $\begin{array}{l}\text { High vs low HR } 1.09 \text { (0.997- } \\
1.20), \mathrm{I}^{2}=59.1 \% \text {. }\end{array}$ & $\mathrm{Xu} 2018^{19}$ \\
\hline \multirow[t]{10}{*}{$\begin{array}{l}\text { Fruits \& } \\
\text { vegetables }\end{array}$} & Root vegetables & 1 & 451,151 & $\begin{array}{l}\text { Prospective cohort } \\
\text { studies up to Sept } 2016\end{array}$ & $\begin{array}{l}\text { Dose-response per } 100 \mathrm{~g} / \text { day } \\
\text { RR } 0.76(0.66-0.88) .\end{array}$ & \multirow[t]{10}{*}{ Aune $2017^{20}$} \\
\hline & $\begin{array}{l}\text { Green leafy } \\
\text { vegetables/salad }\end{array}$ & 7 & 568,725 & $\begin{array}{l}\text { Prospective cohort } \\
\text { studies up to Sept } 2016\end{array}$ & $\begin{array}{l}\text { Dose-response per } 100 \mathrm{~g} / \text { day } \\
\text { RR } 0.78(0.71-0.86), \\
I^{2}=11.1 \% \text {. }\end{array}$ & \\
\hline & Cooked vegetables & 4 & 631,480 & $\begin{array}{l}\text { Prospective cohort } \\
\text { studies up to Sept } 2016\end{array}$ & $\begin{array}{l}\text { Dose-response per } 100 \mathrm{~g} / \text { day } \\
\text { RR } 0.89(0.80-0.99) \\
\mathrm{I}^{2}=94 \% \text {. }\end{array}$ & \\
\hline & $\begin{array}{l}\text { Cruciferous } \\
\text { vegetables }\end{array}$ & 6 & 531,147 & $\begin{array}{l}\text { Prospective cohort } \\
\text { studies up to Sept } 2016\end{array}$ & $\begin{array}{l}\text { Dose-response per } 100 \mathrm{~g} / \text { day } \\
\text { RR } 0.90(0.85-0.95), \\
I^{2}=35.2 \% .\end{array}$ & \\
\hline & Raw vegetables & 2 & 602,120 & $\begin{array}{l}\text { Prospective cohort } \\
\text { studies up to Sept } 2016\end{array}$ & $\begin{array}{l}\text { Dose-response per } 100 \mathrm{~g} / \text { day } \\
\text { RR } 0.91(0.80-1.02) \text {, } \\
\mathrm{I}^{2}=90.8 \% \text {. }\end{array}$ & \\
\hline & Mushrooms & 2 & 495,001 & $\begin{array}{l}\text { Prospective cohort } \\
\text { studies up to Sept } 2016\end{array}$ & $\begin{array}{l}\text { Dose-response per } 100 \mathrm{~g} / \text { day } \\
\text { RR } 0.74(0.46-1.20), \\
\mathrm{I}^{2}=77.7 \% \text {. }\end{array}$ & \\
\hline & $\begin{array}{l}\text { Onion/allium } \\
\text { vegetables }\end{array}$ & 2 & 453,051 & $\begin{array}{l}\text { Prospective cohort } \\
\text { studies up to Sept } 2016\end{array}$ & $\begin{array}{l}\text { Dose-response per } 100 \mathrm{~g} / \text { day } \\
\text { RR } 0.76(0.40-1.46), \\
\mathrm{I}^{2}=50.3 \% .\end{array}$ & \\
\hline & Apples/pears & 3 & 462,571 & $\begin{array}{l}\text { Prospective cohort } \\
\text { studies up to Sept } 2016\end{array}$ & $\begin{array}{l}\text { Dose-response per } 100 \mathrm{~g} / \text { day } \\
\text { RR } 0.80(0.64-1.01) \\
I^{2}=95.3 \% \text {. }\end{array}$ & \\
\hline & Berries & 2 & 461,115 & $\begin{array}{l}\text { Prospective cohort } \\
\text { studies up to Sept } 2016\end{array}$ & $\begin{array}{l}\text { Dose-response per } 100 \mathrm{~g} / \mathrm{day} \\
\text { RR } 0.85(0.70-1.03), \mathrm{I}^{2}=0 \% \text {. }\end{array}$ & \\
\hline & Citrus fruits & 7 & 509,708 & $\begin{array}{l}\text { Prospective cohort } \\
\text { studies up to Sept } 2016\end{array}$ & $\begin{array}{l}\text { Dose-response per } 100 \mathrm{~g} / \text { day } \\
\text { RR } 0.94(0.88-1.00) \text {, } \\
\mathrm{I}^{2}=49.9 \% \text {. }\end{array}$ & \\
\hline
\end{tabular}




\begin{tabular}{|c|c|c|c|c|c|c|}
\hline & Fruit juice & 1 & 109,076 & $\begin{array}{l}\text { Prospective cohort } \\
\text { studies up to Sept } 2016\end{array}$ & $\begin{array}{l}\text { Dose-response per } 100 \mathrm{~g} / \text { day } \\
\text { RR } 0.88(0.84-0.92) .\end{array}$ & \\
\hline & $\begin{array}{l}\text { Non-cruciferous } \\
\text { vegetables }\end{array}$ & 2 & 61,436 & $\begin{array}{l}\text { Prospective cohort } \\
\text { studies up to Sept } 2016\end{array}$ & $\begin{array}{l}\text { Dose-response per } 100 \mathrm{~g} / \text { day } \\
\text { RR } 0.95(0.89-1.02) \\
I^{2}=83.1 \% \text {. }\end{array}$ & \\
\hline & Bananas & 2 & 11,420 & $\begin{array}{l}\text { Prospective cohort } \\
\text { studies up to Sept } 2016\end{array}$ & $\begin{array}{l}\text { Dose-response per } 100 \mathrm{~g} / \text { day } \\
\text { RR } 0.95(0.80-1.14) \\
I^{2}=70.5 \%\end{array}$ & \\
\hline & Tinned fruits & 4 & 147,712 & $\begin{array}{l}\text { Prospective cohort } \\
\text { studies up to Sept } 2016\end{array}$ & $\begin{array}{l}\text { Dose-response per } 100 \mathrm{~g} / \text { day } \\
\text { RR } 1.14(1.07-1.21), \mathrm{I}^{2}=0 \%\end{array}$ & \\
\hline & Potatoes & 5 & 486,865 & $\begin{array}{l}\text { Prospective cohort } \\
\text { studies, up to May } 2018\end{array}$ & $\begin{array}{l}\text { Dose-response per } 150 \mathrm{~g} / \text { day } \\
\text { RR } 0.88(0.69-1.12) \\
\mathrm{I}^{2}=81 \% \text {. }\end{array}$ & $\begin{array}{l}\text { Schwingshackl } \\
2018^{21}\end{array}$ \\
\hline \multirow[t]{6}{*}{ Beverages } & Alcohol & 31 & 844,414 & $\begin{array}{l}\text { Prospective cohort } \\
\text { studies up to Sept } 2009\end{array}$ & $\begin{array}{l}\text { High vs low intake RR } 0.87 \\
(0.83-0.92), \mathrm{I}^{2}=68 \% \text {. }\end{array}$ & $\begin{array}{l}\text { Ronksley } \\
2011^{22}\end{array}$ \\
\hline & Coffee & 16 & 941,247 & $\begin{array}{l}\text { Prospective cohort } \\
\text { studies up to June } 2013\end{array}$ & $\begin{array}{l}\text { Dose-response per cup/day } \\
\text { RR } 0.96(0.94-0.97) . I^{2} \text { not } \\
\text { reported. }\end{array}$ & Je $2014^{23}$ \\
\hline & Green tea & 5 & 205,761 & $\begin{array}{l}\text { Prospective cohort } \\
\text { studies up to Apr } 2015\end{array}$ & $\begin{array}{l}\text { Dose-response per cup/day } \\
\text { RR } 1.01(0.99-1.02), I^{2}=0 \% \text {. }\end{array}$ & \multirow[t]{2}{*}{ Tang $2015^{24}$} \\
\hline & Black tea & 12 & 349,508 & $\begin{array}{l}\text { Prospective cohort } \\
\text { studies up to Apr } 2015\end{array}$ & $\begin{array}{l}\text { Dose-response per cup/day } \\
\text { RR } 0.98(0.86-1.10) \\
I^{2}=84.3 \%\end{array}$ & \\
\hline & $\begin{array}{l}\text { Sugar-sweetened } \\
\text { beverages }\end{array}$ & 3 & 187,402 & $\begin{array}{l}\text { Prospective cohort } \\
\text { studies up to July } 2015\end{array}$ & $\begin{array}{l}\text { High vs low intake RR } 1.03 \\
(0.91-1.18), \mathrm{I}^{2}=75 \% \text {. }\end{array}$ & \multirow[t]{2}{*}{ Narain $2016^{25}$} \\
\hline & $\begin{array}{l}\text { Artificially sweetened } \\
\text { beverages }\end{array}$ & 2 & 173,778 & $\begin{array}{l}\text { Prospective cohort } \\
\text { studies up to July } 2015\end{array}$ & $\begin{array}{l}\text { High vs low intake RR1.09 } \\
(0.92-1.30), \mathrm{I}^{2}=73 \% \text {. }\end{array}$ & \\
\hline \multirow[t]{2}{*}{ Dairy } & Yogurt & 3 & 40,460 & $\begin{array}{l}\text { Prospective cohort } \\
\text { studies up to Sept } 2016\end{array}$ & $\begin{array}{l}\text { Dose-response per } 50 \mathrm{~g} / \text { day } \\
\text { RR } 0.97(0.85-1.11) \text {, } \\
I^{2}=65.8 \% \text {. }\end{array}$ & \multirow[t]{2}{*}{ Guo $2017^{26}$} \\
\hline & Cheese & 11 & 256,091 & $\begin{array}{l}\text { Prospective cohort } \\
\text { studies up to Sept } 2016\end{array}$ & $\begin{array}{l}\text { Dose-response per } 10 \mathrm{~g} / \text { day } \\
\text { RR } 0.99(0.96-1.01)\end{array}$ & \\
\hline
\end{tabular}




\begin{tabular}{|c|c|c|c|c|c|c|}
\hline & & & & & $\mathrm{I}^{2}=93.3 \%$ & \\
\hline & Milk & 10 & 268,570 & $\begin{array}{l}\text { Prospective cohort } \\
\text { studies up to Sept } 2016\end{array}$ & $\begin{array}{l}\text { Dose-response per } 244 \mathrm{~g} / \text { day } \\
\text { RR } 1.00(0.93-1.07), \\
I^{2}=97.4 \% .\end{array}$ & \\
\hline & Butter & 9 & 379,763 & $\begin{array}{l}\text { Prospective cohort } \\
\text { studies up to May } 2015\end{array}$ & $\begin{array}{l}\text { Dose-response per } 14 \mathrm{~g} / \text { day } \\
\text { RR } 1.01(1.00-1.03), \mathrm{I}^{2}=0 \% .\end{array}$ & Pimpin $2018^{7}$ \\
\hline \multirow[t]{5}{*}{ Nuts \& Other } & Nuts & 16 & 819,448 & $\begin{array}{l}\text { Prospective cohort } \\
\text { studies up to July } 2016\end{array}$ & $\begin{array}{l}\text { Dose-response per } 28 \mathrm{~g} / \text { day } \\
\text { RR } 0.78(0.72-0.84) \\
I^{2}=66.0 \%\end{array}$ & \multirow[t]{4}{*}{ Aune $2016^{27}$} \\
\hline & Tree nuts & 4 & 202,751 & $\begin{array}{l}\text { Prospective cohort } \\
\text { studies up to July } 2016\end{array}$ & $\begin{array}{l}\text { Dose-response per } 10 \mathrm{~g} / \text { day } \\
\text { RR } 0.82(0.75-0.90) \\
I^{2}=70.0 \%\end{array}$ & \\
\hline & Peanuts & 5 & 265,252 & $\begin{array}{l}\text { Prospective cohort } \\
\text { studies up to July } 2016\end{array}$ & $\begin{array}{l}\text { Dose-response per } 10 \mathrm{~g} / \text { day } \\
\text { RR } 0.77(0.69-0.86), \\
I^{2}=64.0 \% .\end{array}$ & \\
\hline & Peanut butter & 2 & 83,789 & $\begin{array}{l}\text { Prospective cohort } \\
\text { studies up to July } 2016\end{array}$ & $\begin{array}{l}\text { Dose-response per } 10 \mathrm{~g} / \mathrm{day} \\
\text { RR } 0.94(0.86-1.02), \mathrm{I}^{2}=0 \% .\end{array}$ & \\
\hline & Salt & 7 & 21,515 & $\begin{array}{l}\text { Cohort studies of adults } \\
\text { up to August } 2011 .\end{array}$ & $\begin{array}{l}\text { Dose-response per increase in } \\
\text { sodium intake RR } 1.06(0.94- \\
1.20), \mathrm{I}^{2}=61 \% \text {. }\end{array}$ & Aburto $2013^{28}$ \\
\hline
\end{tabular}


Table 2: Studies that evaluate food items and non-consumption of food items and cardiovascular disease

\begin{tabular}{|c|c|c|c|c|c|c|}
\hline Food group & Food item & $\begin{array}{l}\text { Number } \\
\text { of } \\
\text { studies }\end{array}$ & $\begin{array}{l}\text { Sample } \\
\text { size }\end{array}$ & Inclusion criteria & $\begin{array}{l}\text { Risk estimate for cardiovascular } \\
\text { disease unless otherwise specified }\end{array}$ & Reference \\
\hline \multirow[t]{7}{*}{ Carbohydrate } & $\begin{array}{l}\text { Whole grain } \\
\text { bread }\end{array}$ & 3 & 177,389 & $\begin{array}{l}\text { Prospective cohort } \\
\text { studies up to Apr } 2016\end{array}$ & $\begin{array}{l}\text { Dose-response per } 90 \mathrm{~g} / \text { day RR } 0.87 \\
(0.80-0.95), \mathrm{I}^{2}=0 \%\end{array}$ & \multirow[t]{6}{*}{ Aune $2016^{14}$} \\
\hline & $\begin{array}{l}\text { Whole grain } \\
\text { breakfast } \\
\text { cereal }\end{array}$ & 2 & 206,200 & $\begin{array}{l}\text { Prospective cohort } \\
\text { studies up to Apr } 2016\end{array}$ & $\begin{array}{l}\text { Dose-response per } 30 \mathrm{~g} / \text { day RR } 0.84 \\
(0.78-0.90), \mathrm{I}^{2}=0 \%\end{array}$ & \\
\hline & Bran & 2 & 118,085 & $\begin{array}{l}\text { Prospective cohort } \\
\text { studies up to Apr } 2016\end{array}$ & $\begin{array}{l}\text { Dose-response per } 10 \mathrm{~g} / \text { day RR } 0.85 \\
(0.79-0.90), \mathrm{I}^{2}=0 \% \text {. }\end{array}$ & \\
\hline & Germ & 2 & 118,085 & $\begin{array}{l}\text { Prospective cohort } \\
\text { studies up to Apr } 2016\end{array}$ & $\begin{array}{l}\text { Dose-response per } 2 \mathrm{~g} / \text { day RR } 1.05 \\
(0.96-1.15), \mathrm{I}^{2}=0 \%\end{array}$ & \\
\hline & $\begin{array}{l}\text { Refined } \\
\text { grain }\end{array}$ & 3 & 171,842 & $\begin{array}{l}\text { Prospective cohort } \\
\text { studies up to Apr } 2016\end{array}$ & $\begin{array}{l}\text { Dose-response per } 90 \mathrm{~g} / \text { day RR } 0.98 \\
(0.90,1.06), \mathrm{I}^{2}=56 \%\end{array}$ & \\
\hline & Rice & 3 & 133,393 & $\begin{array}{l}\text { Prospective cohort } \\
\text { studies up to Apr } 2016\end{array}$ & $\begin{array}{l}\text { Dose-response per } 100 \mathrm{~g} / \text { day RR } 0.98 \\
(0.95-1.00), \mathrm{I}^{2}=0 \% \text {. }\end{array}$ & \\
\hline & Fibre & 10 & $1,279,690$ & $\begin{array}{l}\text { Prospective cohort } \\
\text { studies up to Aug } 2013\end{array}$ & $\begin{array}{l}\text { Dose-response per } 7 \mathrm{~g} / \text { day RR } 0.91 \\
(0.88-0.94), \mathrm{I}^{2}=45 \% \text {. }\end{array}$ & $\begin{array}{l}\text { Threapleton } \\
2013^{29}\end{array}$ \\
\hline \multirow[t]{4}{*}{ Meat \& eggs } & Fish & 8 & 331,239 & $\begin{array}{l}\text { Prospective cohort } \\
\text { studies up to Sept } 2016\end{array}$ & $\begin{array}{l}\text { Dose-response per } 20 \mathrm{~g} / \text { day RR } 0.96 \\
(0.94-0.98) \text { for cardiovascular } \\
\text { mortality, } \mathrm{I}^{2}=0 \% \text {. }\end{array}$ & Jayedi $2018^{17}$ \\
\hline & White meat & 5 & $1,197,805$ & $\begin{array}{l}\text { Prospective cohort } \\
\text { studies up to Aug } 2013\end{array}$ & $\begin{array}{l}\text { Dose-response per } 100 \mathrm{~g} / \text { day RR } 1.00 \\
(0.87-1.15) \text { for cardiovascular } \\
\text { mortality, } \mathrm{I}^{2}=36.6 \% \text {. }\end{array}$ & \multirow[t]{3}{*}{ Abete $2014^{18}$} \\
\hline & Red meat & 6 & $1,319,147$ & $\begin{array}{l}\text { Prospective cohort } \\
\text { studies up to Aug } 2013\end{array}$ & $\begin{array}{l}\text { Dose-response per } 100 \mathrm{~g} / \text { day RR } 1.15 \\
(1.05-1.26) \text { for cardiovascular } \\
\text { mortality, } \mathrm{I}^{2}=76.6 \% \text {. }\end{array}$ & \\
\hline & $\begin{array}{l}\text { Processed } \\
\text { meat }\end{array}$ & 6 & $1,186,761$ & $\begin{array}{l}\text { Prospective cohort } \\
\text { studies up to Aug } 2013\end{array}$ & $\begin{array}{l}\text { Dose-response per } 50 \mathrm{~g} / \text { day RR } 1.24 \\
(1.09-1.40) \text { for cardiovascular } \\
\text { mortality, } \mathrm{I}^{2}=76.4 \% \text {. }\end{array}$ & \\
\hline
\end{tabular}




\begin{tabular}{|c|c|c|c|c|c|c|}
\hline & Eggs & 9 & 363,565 & $\begin{array}{l}\text { Prospective cohort } \\
\text { studies up to Mar } 2016\end{array}$ & $\begin{array}{l}\text { High vs low HR } 0.97 \text { (0.90-1.05) for } \\
\text { ischemic heart disease mortality. }\end{array}$ & $\mathrm{Xu} 2018^{19}$ \\
\hline \multirow[t]{14}{*}{$\begin{array}{l}\text { Fruits \& } \\
\text { vegetables }\end{array}$} & $\begin{array}{l}\text { Raw } \\
\text { vegetables }\end{array}$ & 1 & 451,151 & $\begin{array}{l}\text { Prospective cohort } \\
\text { studies up to Sept } 2016\end{array}$ & $\begin{array}{l}\text { Dose-response per } 100 \mathrm{~g} / \text { day RR } 0.86 \\
(0.81-0.90) \text {. }\end{array}$ & \multirow[t]{14}{*}{ Aune $2017^{20}$} \\
\hline & Dried fruit & 1 & 30,458 & $\begin{array}{l}\text { Prospective cohort } \\
\text { studies up to Sept } 2016\end{array}$ & $\begin{array}{l}\text { Dose-response per } 100 \mathrm{~g} / \text { day RR } 0.66 \\
(0.33-1.26) \text {. }\end{array}$ & \\
\hline & Broccoli & 2 & 72,665 & $\begin{array}{l}\text { Prospective cohort } \\
\text { studies up to Sept } 2016\end{array}$ & $\begin{array}{l}\text { Dose-response per } 100 \mathrm{~g} / \text { day RR } 0.75 \\
(0.49-1.14), \mathrm{I}^{2}=0 \% \text {. }\end{array}$ & \\
\hline & $\begin{array}{l}\text { Green leafy } \\
\text { vegetables }\end{array}$ & 5 & 204,508 & $\begin{array}{l}\text { Prospective cohort } \\
\text { studies up to Sept } 2016\end{array}$ & $\begin{array}{l}\text { Dose-response per } 100 \mathrm{~g} / \text { day RR } 0.83 \\
(0.65-1.08), \mathrm{I}^{2}=66.7 \% \text {. }\end{array}$ & \\
\hline & Grapes & 3 & 74,713 & $\begin{array}{l}\text { Prospective cohort } \\
\text { studies up to Sept } 2016\end{array}$ & $\begin{array}{l}\text { Dose-response per } 100 \mathrm{~g} / \text { day RR } 0.83 \\
(0.48-1.45), \mathrm{I}^{2}=66.7 \% \text {. }\end{array}$ & \\
\hline & $\begin{array}{l}\text { Cruciferous } \\
\text { vegetables }\end{array}$ & 9 & 371,431 & $\begin{array}{l}\text { Prospective cohort } \\
\text { studies up to Sept } 2016\end{array}$ & $\begin{array}{l}\text { Dose-response per } 100 \mathrm{~g} / \text { day RR } 0.89 \\
(0.77-1.02), \mathrm{I}^{2}=65.1 \% \text {. }\end{array}$ & \\
\hline & $\begin{array}{l}\text { Non- } \\
\text { cruciferous } \\
\text { vegetables }\end{array}$ & 2 & 134,796 & $\begin{array}{l}\text { Prospective cohort } \\
\text { studies up to Sept } 2016\end{array}$ & $\begin{array}{l}\text { Dose-response per } 100 \mathrm{~g} / \text { day RR } 0.91 \\
(0.82-1.01), \mathrm{I}^{2}=74.5 \% .\end{array}$ & \\
\hline & Citrus fruits & 8 & 239,724 & $\begin{array}{l}\text { Prospective cohort } \\
\text { studies up to Sept } 2016\end{array}$ & $\begin{array}{l}\text { Dose-response per } 100 \mathrm{~g} / \text { day RR } 0.92 \\
(0.84-1.00), \mathrm{I}^{2}=65.8 \% \text {. }\end{array}$ & \\
\hline & $\begin{array}{l}\text { Citrus fruit } \\
\text { juice }\end{array}$ & 2 & 102,368 & $\begin{array}{l}\text { Prospective cohort } \\
\text { studies up to Sept } 2016\end{array}$ & $\begin{array}{l}\text { Dose-response per } 100 \mathrm{~g} / \text { day RR } 0.98 \\
(0.95-1.02), \mathrm{I}^{2}=6.9 \% \text {. }\end{array}$ & \\
\hline & Fruit juice & 2 & 53,989 & $\begin{array}{l}\text { Prospective cohort } \\
\text { studies up to Sept } 2016\end{array}$ & $\begin{array}{l}\text { Dose-response per } 100 \mathrm{~g} / \text { day RR } 0.99 \\
(0.93-1.06), \mathrm{I}^{2}=0 \% \text {. }\end{array}$ & \\
\hline & Apples/pears & 7 & 124,710 & $\begin{array}{l}\text { Prospective cohort } \\
\text { studies up to Sept } 2016\end{array}$ & $\begin{array}{l}\text { Dose-response per } 100 \mathrm{~g} / \text { day RR } 0.92 \\
(0.82-1.03), \mathrm{I}^{2}=46.9 \% \text {. }\end{array}$ & \\
\hline & Tomatoes & 4 & 85,225 & $\begin{array}{l}\text { Prospective cohort } \\
\text { studies up to Sept } 2016\end{array}$ & $\begin{array}{l}\text { Dose-response per } 100 \mathrm{~g} / \text { day RR } 0.92 \\
(0.80-1.07), \mathrm{I}^{2}=52.6 \% \text {. }\end{array}$ & \\
\hline & Carrots & 1 & 9,766 & $\begin{array}{l}\text { Prospective cohort } \\
\text { studies up to Sept } 2016\end{array}$ & $\begin{array}{l}\text { Dose-response per } 100 \mathrm{~g} / \text { day RR } 0.97 \\
(0.72-1.30) \text {. }\end{array}$ & \\
\hline & Strawberries & 1 & 38,176 & $\begin{array}{l}\text { Prospective cohort } \\
\text { studies up to Sept } 2016\end{array}$ & $\begin{array}{l}\text { Dose-response per } 100 \mathrm{~g} / \text { day RR } 1.06 \\
(0.95-1.17) \text {. }\end{array}$ & \\
\hline
\end{tabular}




\begin{tabular}{|c|c|c|c|c|c|c|}
\hline & Tinned fruits & 4 & 106,017 & $\begin{array}{l}\text { Prospective cohort } \\
\text { studies up to Sept } 2016\end{array}$ & $\begin{array}{l}\text { Dose-response per } 100 \mathrm{~g} / \text { day RR } 1.30 \\
(0.81-2.08), \mathrm{I}^{2}=66.0 \% \text {. }\end{array}$ & \\
\hline & Berries & 2 & 40,224 & $\begin{array}{l}\text { Prospective cohort } \\
\text { studies up to Sept } 2016\end{array}$ & $\begin{array}{l}\text { Dose-response per } 100 \mathrm{~g} / \text { day RR } 1.13 \\
(0.88-1.46), \mathrm{I}^{2}=0 \% \text {. }\end{array}$ & \\
\hline & Potatoes & 4 & 202,479 & $\begin{array}{l}\text { Prospective cohort } \\
\text { studies up to Sept } 2016\end{array}$ & $\begin{array}{l}\text { Dose-response per } 100 \mathrm{~g} / \text { day RR } 1.01 \\
(0.97-1.04), \mathrm{I}^{2}=13.4 \% \text {. }\end{array}$ & \\
\hline \multirow[t]{6}{*}{ Beverages } & Alcohol & 21 & $1,184,974$ & $\begin{array}{l}\text { Prospective cohort } \\
\text { studies up to Sept } 2009\end{array}$ & $\begin{array}{l}\text { High vs low intake RR } 0.75(0.70- \\
0.80) \text { for cardiovascular mortality, } \\
\mathrm{I}^{2}=72.2 \% \text {. }\end{array}$ & $\begin{array}{l}\text { Ronksley } \\
2011^{22}\end{array}$ \\
\hline & Coffee & 16 & $1,029,237$ & $\begin{array}{l}\text { Prospective cohort } \\
\text { studies up to Jan } 2013\end{array}$ & $\begin{array}{l}\text { Dose-response per cup/day RR } 0.98 \\
(0.95-1.00) \text { for cardiovascular } \\
\text { mortality, } \mathrm{I}^{2}=87.8 \% \text {. }\end{array}$ & $\begin{array}{l}\text { Malerba } \\
2013^{30}\end{array}$ \\
\hline & Green tea & 5 & 197,957 & $\begin{array}{l}\text { Prospective cohort } \\
\text { studies up to Apr } 2015\end{array}$ & $\begin{array}{l}\text { Dose-response per cup/day RR } 0.95 \\
(0.90-1.00) \text { for cardiovascular } \\
\text { mortality, } \mathrm{I}^{2}=83.8 \% \text {. }\end{array}$ & \multirow[t]{2}{*}{ Tang $2015^{24}$} \\
\hline & Black tea & 7 & 162,230 & $\begin{array}{l}\text { Prospective cohort } \\
\text { studies up to Apr } 2015\end{array}$ & $\begin{array}{l}\text { Dose-response per cup/day RR } 0.92 \\
(0.85-0.99) \text { for cardiovascular } \\
\text { mortality, } \mathrm{I}^{2}=75.6 \% \text {. }\end{array}$ & \\
\hline & $\begin{array}{l}\text { Sugar- } \\
\text { sweetened } \\
\text { beverages }\end{array}$ & 1 & 2,564 & $\begin{array}{l}\text { Prospective cohort } \\
\text { studies up to July } 2015\end{array}$ & $\begin{array}{l}\text { High vs low intake RR } 1.00(0.98- \\
1.02) \text { for vascular event. }\end{array}$ & \multirow[t]{2}{*}{$\begin{array}{l}\text { Narain } \\
2016^{25}\end{array}$} \\
\hline & $\begin{array}{l}\text { Artificially } \\
\text { sweetened } \\
\text { beverages }\end{array}$ & 1 & 2,564 & $\begin{array}{l}\text { Prospective cohort } \\
\text { studies up to July } 2015\end{array}$ & $\begin{array}{l}\text { High vs low intake RR } 1.02(1.00- \\
1.05) \text { for vascular event. }\end{array}$ & \\
\hline \multirow[t]{4}{*}{ Dairy } & Yogurt & 3 & 36,624 & $\begin{array}{l}\text { Prospective cohort } \\
\text { studies up to Sept } 2016\end{array}$ & $\begin{array}{l}\text { Dose-response per 50g/day RR } 1.03 \\
(0.97-1.09), \mathrm{I}^{2}=0 \% .\end{array}$ & \multirow[t]{3}{*}{ Guo $2017^{26}$} \\
\hline & Cheese & 9 & 234,447 & $\begin{array}{l}\text { Prospective cohort } \\
\text { studies up to Sept } 2016\end{array}$ & $\begin{array}{l}\text { Dose-response per } 10 \mathrm{~g} / \mathrm{day} \mathrm{RR} 0.98 \\
(0.95-1.00), \mathrm{I}^{2}=82.6 \% \text {. }\end{array}$ & \\
\hline & Milk & 9 & 249,779 & $\begin{array}{l}\text { Prospective cohort } \\
\text { studies up to Sept } 2016\end{array}$ & $\begin{array}{l}\text { Dose-response per } 244 \mathrm{~g} / \text { day RR } 1.01 \\
(0.93-1.10), \mathrm{I}^{2}=92.4 \% \text {. }\end{array}$ & \\
\hline & Butter & 2 & 147,297 & $\begin{array}{l}\text { Prospective cohort } \\
\text { studies up to May } 2015\end{array}$ & $\begin{array}{l}\text { Dose-response per } 14 \mathrm{~g} / \text { day RR } 0.99 \\
(0.96-1.02), \mathrm{I}^{2}=0 \% .\end{array}$ & Pimpin $2018^{7}$ \\
\hline
\end{tabular}




\begin{tabular}{|c|c|c|c|c|c|c|}
\hline \multirow[t]{9}{*}{ Nuts \& Other } & Nuts & 12 & 376,228 & $\begin{array}{l}\text { Prospective cohort } \\
\text { studies up to July } 2016\end{array}$ & $\begin{array}{l}\text { Dose-response per } 28 \mathrm{~g} / \text { day RR } 0.79 \\
(0.70-0.88), \mathrm{I}^{2}=59.6 \% \text {. }\end{array}$ & \multirow[t]{3}{*}{ Aune $2016^{27}$} \\
\hline & Tree nuts & 3 & 130,987 & $\begin{array}{l}\text { Prospective cohort } \\
\text { studies up to July } 2016\end{array}$ & $\begin{array}{l}\text { Dose-response per } 10 \mathrm{~g} / \text { day RR } \\
0.75(0.67-0.84), \mathrm{I}^{2}=0 \% \text {. }\end{array}$ & \\
\hline & Peanuts & 5 & 265,252 & $\begin{array}{l}\text { Prospective cohort } \\
\text { studies up to July } 2016\end{array}$ & $\begin{array}{l}\text { Dose-response per } 10 \mathrm{~g} / \text { day RR } 0.64 \\
(0.50-0.81), \mathrm{I}^{2}=77.0 \% \text {. }\end{array}$ & \\
\hline & Salt & 9 & 46,483 & $\begin{array}{l}\text { Cohort studies of adults } \\
\text { up to August } 2011 .\end{array}$ & $\begin{array}{l}\text { Dose-response per increase in } \\
\text { sodium intake } 1.12(0.93-1.34) \text {, } \\
\mathrm{I}^{2}=61 \% \text {. }\end{array}$ & $\begin{array}{l}\text { Aburto } \\
2013^{28}\end{array}$ \\
\hline & Olive oil & 9 & 476,714 & $\begin{array}{l}\text { Case-control, prospective } \\
\text { studies and randomized } \\
\text { trials up to Dec } 2013\end{array}$ & $\begin{array}{l}\text { Dose-response per } 25 \mathrm{~g} / \text { day RR } 0.82 \\
(0.70-0.96), \mathrm{I}^{2}=77 \% \text {. }\end{array}$ & $\begin{array}{l}\text { Martinez- } \\
\text { Gonzalez } \\
2014^{31}\end{array}$ \\
\hline & Soy & 20 & 718,279 & $\begin{array}{l}\text { Prospective cohort and } \\
\text { case control studies up to } \\
\text { Feb } 2016\end{array}$ & $\begin{array}{l}\text { High vs low RR } 0.83(0.75-0.93) \text {, } \\
\mathrm{I}^{2}=71.4 \% \text {. }\end{array}$ & \multirow[t]{3}{*}{ Yan $2017^{8}$} \\
\hline & Tofu & 4 & 260,607 & $\begin{array}{l}\text { Prospective cohort and } \\
\text { case control studies up to } \\
\text { Feb } 2016\end{array}$ & $\begin{array}{l}\text { High vs low RR } 0.80 \text { (0.64-1.00), } \\
\mathrm{I}^{2}=75.1 \% \text {. }\end{array}$ & \\
\hline & Miso & 2 & 42,371 & $\begin{array}{l}\text { Prospective cohort and } \\
\text { case control studies up to } \\
\text { Feb } 2016\end{array}$ & $\begin{array}{l}\text { High vs low RR } 0.82 \text { (0.64-1.06), } \\
\mathrm{I}^{2}=29.8 \% \text {. }\end{array}$ & \\
\hline & Chocolate & 12 & 369,599 & $\begin{array}{l}\text { Prospective cohort } \\
\text { studies up to Jun } 2018\end{array}$ & $\begin{array}{l}\text { Dose-response per } 20 \mathrm{~g} / \text { week } 0.982 \\
(0.972-0.992), \mathrm{I}^{2}=50.4 \% \text {. }\end{array}$ & Ren $2018^{32}$ \\
\hline
\end{tabular}


Supplementary Figure 1: Study selection process

$$
\begin{aligned}
& \text { 3,011 studies retrieved from the search } \\
& \text { described in the methods and presented } \\
& \text { in Supplementary Table } 1 .
\end{aligned}
$$

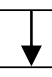

341 reviews or studies reviewed in detail for potential inclusion.

16 reviews for all-cause mortality.

17 reviews for cardiovascular disease. 
Supplementary Table 1: Food categories, food components and search results

\begin{tabular}{|c|c|c|}
\hline Food Category & Food component & Search results \\
\hline \multirow[t]{7}{*}{ Fats and Oil } & Olive oil & 35 \\
\hline & Palm oil & 4 \\
\hline & Sunflower oil & 0 \\
\hline & Sesame oil & 0 \\
\hline & Peanut oil & 0 \\
\hline & Butter & 16 \\
\hline & Margarine & 5 \\
\hline \multirow[t]{4}{*}{ Dairy } & Milk & 140 \\
\hline & Yogurt & 11 \\
\hline & Ice cream & 2 \\
\hline & Cheese & 15 \\
\hline \multirow{8}{*}{$\begin{array}{l}\text { Meat, poultry and } \\
\text { beans }\end{array}$} & Pork or pig & 124 \\
\hline & Beef or cow & 84 \\
\hline & Lamb or sheep & 53 \\
\hline & Chicken & 26 (39 with poultry) \\
\hline & Turkey & 257 \\
\hline & Duck & 4 \\
\hline & Beans or legumes or pulses & 361 \\
\hline & Tofu or soybean & 39 \\
\hline \multirow[t]{13}{*}{ Fish and seafood } & Salmon & 32 \\
\hline & Tuna & 2 \\
\hline & Cod or bass & 64 \\
\hline & Catfish & 0 \\
\hline & Mackerel & 0 \\
\hline & Anchovy & 0 \\
\hline & Herring & 4 \\
\hline & Shark & 1 \\
\hline & Shrimp or prawn & 1 \\
\hline & Squid or octopus & 4 \\
\hline & $\begin{array}{l}\text { Shellfish or oyster or mussel or scallop } \\
\text { or clams }\end{array}$ & 6 \\
\hline & Crab or lobster & 3 \\
\hline & Mussel & 0 \\
\hline Eggs & Eggs & 37 (51 egg) \\
\hline \multirow[t]{10}{*}{ Nuts } & Almond & 9 \\
\hline & Chestnuts & 0 (26 chestnut) \\
\hline & Hazelnuts & 1 \\
\hline & Walnuts & 6 \\
\hline & Cashews & 0 \\
\hline & Pistachios & 2 \\
\hline & Pine nuts & 0 \\
\hline & Brazil nuts & 0 \\
\hline & Macadamia nuts & 1 \\
\hline & Peanuts & 5 \\
\hline \multirow[t]{3}{*}{ Vegetables } & Broccoli & 5 \\
\hline & Cabbage & 2 \\
\hline & Carrots & 4 \\
\hline
\end{tabular}




\begin{tabular}{|c|c|c|}
\hline & Celery & 0 \\
\hline & Corn & 14 \\
\hline & Lettuce & 0 \\
\hline & Peas & 0 \\
\hline & Spinach & 1 \\
\hline & Cauliflower & 3 \\
\hline & Chickpea & 0 \\
\hline & Asparagus & 1 \\
\hline & Garlic & 23 \\
\hline & Onion & 3 \\
\hline & Ginger & 4 \\
\hline & Seaweed & 1 \\
\hline \multirow[t]{30}{*}{ Fruit } & Apple & 23 \\
\hline & Bananas & 9 \\
\hline & Blueberry & 0 \\
\hline & Blackberry & 2 \\
\hline & Cherry & 22 \\
\hline & Coconut & 2 \\
\hline & Cranberry & 5 \\
\hline & Grapes & 6 \\
\hline & Figs & 2 \\
\hline & Dates & 144 \\
\hline & Kiwifruit & 0 \\
\hline & Mango & 1 \\
\hline & Lychee & 0 \\
\hline & Olive & 55 \\
\hline & Peach & 6 \\
\hline & Pear & 2 \\
\hline & Plum & 1 \\
\hline & Pineapple & 0 \\
\hline & Raspberry & 0 \\
\hline & Strawberry & 0 \\
\hline & Orange & 106 \\
\hline & Lemon & 17 \\
\hline & Avocado & 3 \\
\hline & Pepper & 0 \\
\hline & Melons & 3 \\
\hline & Cucumber & 0 \\
\hline & Pumpkins & 0 \\
\hline & Squash & 0 \\
\hline & Tomato & 6 \\
\hline & Courgettes or zucchini & 1 \\
\hline \multirow{7}{*}{$\begin{array}{l}\text { Carbohydrate and } \\
\text { grains }\end{array}$} & Bread & 6 \\
\hline & Rice & 123 \\
\hline & Cereal & 37 \\
\hline & Pasta & 3 \\
\hline & Fibre or fibre & 177 \\
\hline & Potatoes & 6 \\
\hline & Noodles & 0 \\
\hline Drinks and & Coffee & 50 \\
\hline
\end{tabular}




\begin{tabular}{|l|l|l|}
\hline \multirow{4}{*}{ beverages } & Tea & 54 \\
\cline { 2 - 3 } & Wine & 38 \\
\cline { 2 - 3 } & Beer & 54 \\
\cline { 2 - 3 } & Spirits or vodka or gin or whisky or rum & 35 \\
\cline { 2 - 3 } & Soft drinks & 3 \\
\hline \multirow{5}{*}{ Snacks and sweets } & Crisps & 0 \\
\cline { 2 - 3 } & Chocolate & 19 \\
\cline { 2 - 3 } & Confectionary or sweets & 21 \\
\cline { 2 - 3 } & Biscuits or cookies & 0 \\
\hline \multirow{2}{*}{$\begin{array}{l}\text { Sauces and } \\
\text { condiments }\end{array}$} & Sauces and condiments & 1 \\
\hline Salt & & \\
\hline Fungus & Salt & 495 \\
\hline
\end{tabular}

Search took place on 13 August 2018. 
Supplementary Table 2: Grading of meta-analyses based on Grosso et al.

\begin{tabular}{|l|l|l|}
\hline Level of evidence & Level & Definition \\
\hline Convincing & $\begin{array}{l}\text { 1a (high) } \\
\text { 1b (low) }\end{array}$ & $\begin{array}{l}\text { Concordance of meta-analysis of RCTs and meta-analysis of } \\
\text { observational studies. } \\
\text { Meta-analysis of RCTs with contrary results to observational } \\
\text { studies. }\end{array}$ \\
\hline Probable & 2 & Meta-analysis of prospective studies with no heterogeneity. \\
\hline Possible & 3 & $\begin{array}{l}\text { Meta-analysis of prospective or retrospective study lacking } \\
\text { information on statistic heterogeneity or with } ~^{2}>30 \% .\end{array}$ \\
\hline Limited/contrasting & 4 & Limited studies included in meta-analysis $(\mathrm{n} \leq 3)$. \\
\hline
\end{tabular}

Grosso G, Godos J, Alvano F, Giovannucci EL. Coffee, caffeine, and health outcome: an umbrella review. Ann Rev Nutr 2017;37:131-156. 
Supplementary Table 3: Quality assessments in the included systematic reviews and meta-analyses

\begin{tabular}{|c|c|c|c|}
\hline Review ID & $\begin{array}{l}\text { Dietary } \\
\text { component }\end{array}$ & Assessment method & Quality assessment \\
\hline Aune $2016^{14}$ & Grain & $\begin{array}{l}\text { Newcastle-Ottawa } \\
\text { scale (0-9). }\end{array}$ & $\begin{array}{l}\text { Average quality assessment score for CVD was } \\
7.7 / 9 \text { and all-cause death } 7.9 / 9 \text {. }\end{array}$ \\
\hline Saneei $2017^{15}$ & Rice & $\begin{array}{l}\text { Hu et al score (out of } \\
15 \text { ). }\end{array}$ & $\begin{array}{l}\text { Average quality assessment score for mortality } \\
\text { was } 10.3 / 15 \text {. }\end{array}$ \\
\hline Yang $2015^{16}$ & Fibre & No quality score used. & Not performed. \\
\hline Jayedi $2018^{17}$ & Fish & $\begin{array}{l}\text { Newcastle-Ottawa } \\
\text { scale (0-9). }\end{array}$ & $\begin{array}{l}\text { Average quality assessment score overall was } \\
7.5 / 9 \text {. }\end{array}$ \\
\hline Abete $2014^{28}$ & Meat & No quality score used. & Not performed. \\
\hline Xu $2018^{19}$ & Eggs & No quality score used. & Not performed. \\
\hline Aune $2017^{20}$ & $\begin{array}{l}\text { Fruits and } \\
\text { vegetables }\end{array}$ & $\begin{array}{l}\text { Newcastle-Ottawa } \\
\text { scale (0-9). }\end{array}$ & $\begin{array}{l}\text { Quality assessment scores for CVD was 12/13 for } \\
\text { fruits/vegetables, 15/17 for fruits and 12/14 for } \\
\text { vegetables. For all-cause mortality, it was } 14 / 15 \\
\text { for fruits/vegetables, } 20 / 27 \text { for fruits and 19/22 } \\
\text { for vegetables. }\end{array}$ \\
\hline $\begin{array}{l}\text { Schwingshackl } \\
2018^{21}\end{array}$ & Potatoes & $\begin{array}{l}\text { NutriGrade scoring } \\
\text { system (out of } 10 \text { but } \\
\text { graded as very low (0- } \\
3 \text { ), low (4-5), } \\
\text { moderate (6-7) and } \\
\text { high ( } \geq 8) \text {. }\end{array}$ & $\begin{array}{l}\text { Average quality for all-cause mortality was low } \\
\text { and CHD was low. }\end{array}$ \\
\hline $\begin{array}{l}\text { Ronksley } \\
2011^{22}\end{array}$ & Alcohol & $\begin{array}{l}2 \text { criteria assessed } \\
\text { based on Egger et al } \\
\text { and Laupacis et al. }\end{array}$ & $\begin{array}{l}85 \% \text { of studies had }>5 \text { years follow up and } 90 \% \text { of } \\
\text { studies adjusted for basic demographic } \\
\text { information. }\end{array}$ \\
\hline Je $2014^{23}$ & Coffee & $\begin{array}{l}\text { Adjustments for } \\
\text { potential confounders } \\
\text { only factor } \\
\text { considered. }\end{array}$ & All studies adjusted for covariates. \\
\hline Tang $2015^{24}$ & Tea & $\begin{array}{l}\text { Newcastle-Ottawa } \\
\text { scale (0-9). }\end{array}$ & $\begin{array}{l}\text { Average quality assessment score overall was } \\
6.1 / 9 \text {. }\end{array}$ \\
\hline Narain $2016^{25}$ & Soft drink & 5 areas assessed. & $\begin{array}{l}\text { Average quality assessment score overall was } \\
3.6 / 5 \text {. }\end{array}$ \\
\hline Guo $2017^{26}$ & Milk & $\begin{array}{l}\text { Newcastle-Ottawa } \\
\text { quality assessment } \\
\text { scale (0-9). }\end{array}$ & $\begin{array}{l}\text { Average quality assessment score overall was } \\
7.9 / 9 .\end{array}$ \\
\hline Pimpin $2018^{7}$ & Butter & $\begin{array}{l}\text { Adapted Newcastle- } \\
\text { Ottawa quality scale } \\
(0-5) \text {. }\end{array}$ & Average quality assessment score overall 4.6/5. \\
\hline Aune $2016^{27}$ & Nuts & $\begin{array}{l}\text { Newcastle-Ottawa } \\
\text { scale (0-9). }\end{array}$ & $\begin{array}{l}\text { Average quality assessment score for CVD was } \\
7.6 / 9 \text { and all-cause death } 7.3 / 9 \text {. }\end{array}$ \\
\hline Aburto $2013^{28}$ & Salt & $\begin{array}{l}\text { GRADE methodology } \\
\text { used to assess quality. }\end{array}$ & $\begin{array}{l}\text { Quality of the evidence was very low to } \\
\text { moderate for CVD, very low to low for CHD and } \\
\text { very low for all-cause mortality. }\end{array}$ \\
\hline $\begin{array}{l}\text { Threapleton } \\
2013^{29}\end{array}$ & Fibre & $\begin{array}{l}\text { Newcastle-Ottawa } \\
\text { scale (0-9). }\end{array}$ & Average quality assessment score overall 7.2/9. \\
\hline Malerba $2013^{30}$ & Coffee & No quality score used. & Not performed. \\
\hline $\begin{array}{l}\text { Martinez- } \\
\text { Gonzalez } \\
2014^{31}\end{array}$ & Olive oil & $\begin{array}{l}\text { Newcastle-Ottawa } \\
\text { scale }(0-9) \text {. }\end{array}$ & Average quality assessment score overall 7.8/9. \\
\hline Yan $2017^{8}$ & Soy & $\begin{array}{l}\text { Newcastle-Ottawa } \\
\text { scale (0-9). }\end{array}$ & Average quality assessment score overall 7.7/9. \\
\hline Ren $2018^{32}$ & Chocolate & Newcastle-Ottawa & Average quality assessment score overall 8.4/9. \\
\hline
\end{tabular}




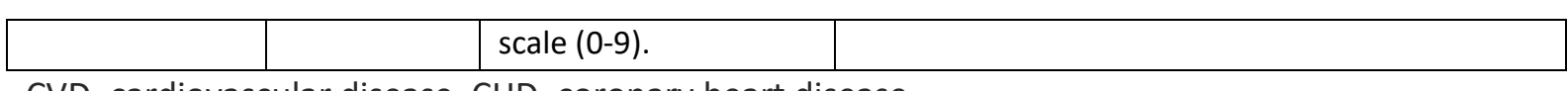

$\mathrm{CVD}=$ cardiovascular disease, $\mathrm{CHD}=$ coronary heart disease 
Supplementary Table 4: Grading the quality of the evidence for each food component

\begin{tabular}{|c|c|c|c|c|c|}
\hline Food group & Food item & $\begin{array}{l}\text { Grade for } \\
\text { mortality }\end{array}$ & Reason & $\begin{array}{l}\text { Grade for } \\
\text { CVD }\end{array}$ & Reason \\
\hline \multirow[t]{9}{*}{ Carbohydrate } & Whole grain bread & $\begin{array}{l}\text { Level } 4 \\
\text { limited }\end{array}$ & Fewer than 4 studies. & $\begin{array}{l}\text { Level } 4 \\
\text { limited }\end{array}$ & Fewer than 4 studies. \\
\hline & Pasta & $\begin{array}{l}\text { Level } 4 \\
\text { limited }\end{array}$ & Fewer than 4 studies. & - & - \\
\hline & Whole grain breakfast cereal & $\begin{array}{l}\text { Level } 4 \\
\text { limited }\end{array}$ & Fewer than 4 studies. & $\begin{array}{l}\text { Level } 4 \\
\text { limited }\end{array}$ & Fewer than 4 studies. \\
\hline & Oats/oatmeal & $\begin{array}{l}\text { Level } 4 \\
\text { limited }\end{array}$ & Fewer than 4 studies. & $\begin{array}{l}\text { Level } 4 \\
\text { limited }\end{array}$ & Fewer than 4 studies. \\
\hline & Refined grain & $\begin{array}{l}\text { Level } 2 \\
\text { probable }\end{array}$ & $\begin{array}{l}4 \text { prospective studies with } \\
I^{2}=20 \% .\end{array}$ & - & - \\
\hline & Bran & - & - & $\begin{array}{l}\text { Level } 4 \\
\text { limited }\end{array}$ & Fewer than 4 studies. \\
\hline & Germ & - & - & $\begin{array}{l}\text { Level } 4 \\
\text { limited }\end{array}$ & Fewer than 4 studies. \\
\hline & Rice & $\begin{array}{l}\text { Level } 3 \\
\text { possible }\end{array}$ & 5 cohort studies with $\mathrm{I}^{2}=39.4 \%$. & $\begin{array}{l}\text { Level } 4 \\
\text { limited }\end{array}$ & Fewer than 4 studies. \\
\hline & Fibre & $\begin{array}{l}\text { Level } 3 \\
\text { possible }\end{array}$ & $\begin{array}{l}5 \text { prospective studies with } \\
I^{2}=77.2 \% .\end{array}$ & $\begin{array}{l}\text { Level } 3 \\
\text { possible }\end{array}$ & 10 prospective studies with $\mathrm{I}^{2}=45 \%$. \\
\hline \multirow[t]{5}{*}{ Meat \& eggs } & Fish & $\begin{array}{l}\text { Level } 3 \\
\text { possible }\end{array}$ & $\begin{array}{l}14 \text { prospective studies with } \\
I^{2}=81.9 \% .\end{array}$ & $\begin{array}{l}\text { Level } 2 \\
\text { possible }\end{array}$ & 8 prospective studies with $\mathrm{I}^{2}=0 \%$. \\
\hline & White meat & $\begin{array}{l}\text { Level } 3 \\
\text { possible }\end{array}$ & $\begin{array}{l}5 \text { prospective studies with } \\
I^{2}=92.1 \% .\end{array}$ & $\begin{array}{l}\text { Level } 3 \\
\text { possible }\end{array}$ & 5 prospective studies with $\mathrm{I}^{2}=36.6 \%$. \\
\hline & Red meat & $\begin{array}{l}\text { Level } 3 \\
\text { possible }\end{array}$ & $\begin{array}{l}6 \text { prospective studies with } \\
I^{2}=95 \% .\end{array}$ & $\begin{array}{l}\text { Level } 3 \\
\text { possible }\end{array}$ & 6 prospective studies with $\mathrm{I}^{2}=76.6 \%$. \\
\hline & Processed meat & $\begin{array}{l}\text { Level } 3 \\
\text { possible }\end{array}$ & $\begin{array}{l}5 \text { prospective studies with } \\
\mathrm{I}^{2}=95.7 \% \text {. }\end{array}$ & $\begin{array}{l}\text { Level } 3 \\
\text { possible }\end{array}$ & 6 prospective studies with $I^{2}=76.4 \%$. \\
\hline & Eggs & $\begin{array}{l}\text { Level } 3 \\
\text { possible }\end{array}$ & $\begin{array}{l}4 \text { prospective studies with } \\
I^{2}=59.1 \% .\end{array}$ & $\begin{array}{l}\text { Level } 3 \\
\text { possible }\end{array}$ & $\begin{array}{l}9 \text { prospective studies with } I^{2} \text { not } \\
\text { reported. }\end{array}$ \\
\hline
\end{tabular}




\begin{tabular}{|c|c|c|c|c|c|}
\hline \multirow[t]{16}{*}{ Fruits \& vegetables } & Root vegetables & $\begin{array}{l}\text { Level } 4 \\
\text { limited }\end{array}$ & Fewer than 4 studies. & - & - \\
\hline & Green leafy vegetables/salad & $\begin{array}{l}\text { Level } 2 \\
\text { probable. }\end{array}$ & $\begin{array}{l}7 \text { prospective studies with } \\
\mathrm{I}^{2}=11.1 \% \text {. }\end{array}$ & $\begin{array}{l}\text { Level } 3 \\
\text { possible }\end{array}$ & 5 prospective studies with $\mathrm{I}^{2}=66.7 \%$ \\
\hline & Cooked vegetables & $\begin{array}{l}\text { Level } 3 \\
\text { possible }\end{array}$ & $\begin{array}{l}4 \text { prospective studies with } \\
\mathrm{I}^{2}=94 \% .\end{array}$ & - & - \\
\hline & Cruciferous vegetables & $\begin{array}{l}\text { Level } 3 \\
\text { possible }\end{array}$ & $\begin{array}{l}6 \text { prospective studies with } \\
\mathrm{I}^{2}=35.2 \% .\end{array}$ & $\begin{array}{l}\text { Level } 3 \\
\text { possible }\end{array}$ & 9 prospective studies with $\mathrm{I}^{2}=65.1 \%$. \\
\hline & Raw vegetables & $\begin{array}{l}\text { Level } 4 \\
\text { limited }\end{array}$ & Fewer than 4 studies. & $\begin{array}{l}\text { Level } 4 \\
\text { limited }\end{array}$ & Fewer than 4 studies. \\
\hline & Mushrooms & $\begin{array}{l}\text { Level } 4 \\
\text { limited }\end{array}$ & Fewer than 4 studies. & - & - \\
\hline & Onion/allium vegetables & $\begin{array}{l}\text { Level } 4 \\
\text { limited } \\
\end{array}$ & Fewer than 4 studies. & - & - \\
\hline & Apples/pears & $\begin{array}{l}\text { Level } 4 \\
\text { limited }\end{array}$ & Fewer than 4 studies. & $\begin{array}{l}\text { Level } 3 \\
\text { possible }\end{array}$ & 7 prospective studies with $I^{2}=46.9 \%$ \\
\hline & Berries & $\begin{array}{l}\text { Level } 4 \\
\text { limited }\end{array}$ & Fewer than 4 studies. & $\begin{array}{l}\text { Level } 4 \\
\text { limited }\end{array}$ & Fewer than 4 studies. \\
\hline & Citrus fruits & $\begin{array}{l}\text { Level } 3 \\
\text { possible }\end{array}$ & $\begin{array}{l}7 \text { prospective studies with } \\
\mathrm{I}^{2}=49.9 \% \text {. }\end{array}$ & $\begin{array}{l}\text { Level } 3 \\
\text { possible }\end{array}$ & 8 prospective studies with $\mathrm{I}^{2}=65.8 \%$. \\
\hline & Fruit juice & $\begin{array}{l}\text { Level } 4 \\
\text { limited }\end{array}$ & Fewer than 4 studies. & $\begin{array}{l}\text { Level } 4 \\
\text { limited }\end{array}$ & Fewer than 4 studies. \\
\hline & Non-cruciferous vegetables & $\begin{array}{l}\text { Level } 4 \\
\text { limited } \\
\end{array}$ & Fewer than 4 studies. & $\begin{array}{l}\text { Level } 4 \\
\text { limited }\end{array}$ & Fewer than 4 studies. \\
\hline & Bananas & $\begin{array}{l}\text { Level } 4 \\
\text { limited }\end{array}$ & Fewer than 4 studies. & - & - \\
\hline & Tinned fruits & $\begin{array}{l}\text { Level } 2 \\
\text { probable }\end{array}$ & $\begin{array}{l}4 \text { prospective studies with } \\
\mathrm{I}^{2}=0 \% .\end{array}$ & $\begin{array}{l}\text { Level } 3 \\
\text { possible }\end{array}$ & 4 prospective studies with $I^{2}=66.0 \%$ \\
\hline & Carrots & - & - & $\begin{array}{l}\text { Level } 4 \\
\text { limited }\end{array}$ & Fewer than 4 studies. \\
\hline & Strawberries & - & - & $\begin{array}{l}\text { Level } 4 \\
\text { limited }\end{array}$ & Fewer than 4 studies. \\
\hline
\end{tabular}




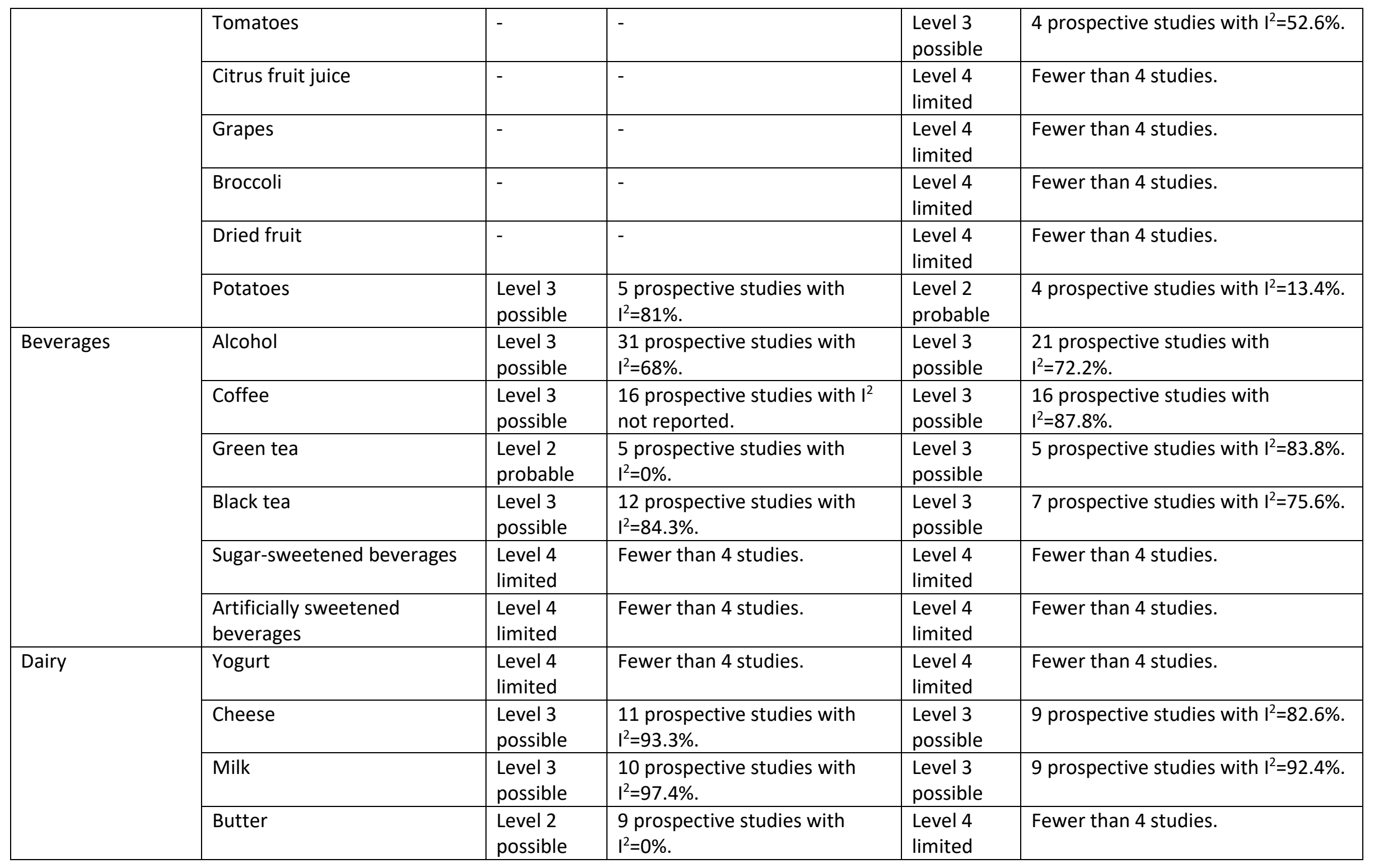




\begin{tabular}{|c|c|c|c|c|c|}
\hline \multirow[t]{10}{*}{ Nuts \& Other } & Nuts & $\begin{array}{l}\text { Level } 3 \\
\text { possible }\end{array}$ & $\begin{array}{l}16 \text { prospective studies with } \\
I^{2}=66.0 \% .\end{array}$ & $\begin{array}{l}\text { Level } 3 \\
\text { possible }\end{array}$ & $\begin{array}{l}12 \text { prospective studies with } \\
I^{2}=59.6 \% .\end{array}$ \\
\hline & Tree nuts & $\begin{array}{l}\text { Level } 3 \\
\text { possible }\end{array}$ & $\begin{array}{l}4 \text { prospective studies with } \\
I^{2}=70.0 \% \text {. }\end{array}$ & $\begin{array}{l}\text { Level } 4 \\
\text { limited }\end{array}$ & Fewer than 4 studies. \\
\hline & Peanuts & $\begin{array}{l}\text { Level } 3 \\
\text { possible }\end{array}$ & $\begin{array}{l}5 \text { prospective studies with } \\
\mathrm{I}^{2}=64.0 \% .\end{array}$ & $\begin{array}{l}\text { Level } 3 \\
\text { possible }\end{array}$ & 5 prospective studies with $\left.\right|^{2}=77.0-\%$. \\
\hline & Peanut butter & $\begin{array}{l}\text { Level } 4 \\
\text { limited }\end{array}$ & Fewer than 4 studies. & - & - \\
\hline & Salt & $\begin{array}{l}\text { Level } 3 \\
\text { possible }\end{array}$ & $\begin{array}{l}7 \text { prospective studies with } \\
I^{2}=61 \% \text {. }\end{array}$ & $\begin{array}{l}\text { Level } 3 \\
\text { possible }\end{array}$ & 9 prospective studies with $\mathrm{I}^{2}=61 \%$. \\
\hline & Olive oil & - & - & $\begin{array}{l}\text { Level } 3 \\
\text { possible }\end{array}$ & 9 prospective studies with $\mathrm{I}^{2}=77 \%$. \\
\hline & Soy & - & - & $\begin{array}{l}\text { Level } 3 \\
\text { possible }\end{array}$ & $\begin{array}{l}20 \text { prospective studies with } \\
\mathrm{I}^{2}=71.4 \% \text {. }\end{array}$ \\
\hline & Tofu & - & - & $\begin{array}{l}\text { Level } 3 \\
\text { possible }\end{array}$ & 4 prospective studies with $\mathrm{I}^{2}=75.1 \%$. \\
\hline & Miso & - & - & $\begin{array}{l}\text { Level } 4 \\
\text { limited }\end{array}$ & Fever than 4 studies. \\
\hline & Chocolate & - & - & $\begin{array}{l}\text { Level } 3 \\
\text { possible }\end{array}$ & 12 prospective studies with $I^{2}=61 \%$. \\
\hline
\end{tabular}


Supplementary Table 5: Consideration of sex differences among included studies

\begin{tabular}{|c|c|c|}
\hline Review ID & $\begin{array}{l}\text { Dietary } \\
\text { component }\end{array}$ & Consideration of sex differences among included studies \\
\hline Aune $2016^{14}$ & Grain & $\begin{array}{l}\text { The authors state that there was little evidence of heterogeneity between subgroups in } \\
\text { subgroup and meta-regression stratified by sex. }\end{array}$ \\
\hline Saneei $2017^{15}$ & Rice & Risk of mortality in men RR 0.87 (0.81-0.94) and in women RR 1.08 (0.97-1.19). \\
\hline Yang $2015^{16}$ & Fibre & $\begin{array}{l}\text { For top vs bottom tertile, risk of mortality in men RR } 0.80(0.76-0.85) \text { and in women RR } 0.83 \\
(0.79-0.86) \text {. }\end{array}$ \\
\hline Jayedi $2018^{17}$ & Fish & Risk of mortality in men was RR $0.99(0.96-1.02)$ and in women it was RR $0.98(0.95-1.00)$ \\
\hline Abete $2014^{18}$ & Meat & $\begin{array}{l}\text { Risk of mortality in men for red meat RR } 1.21 \text { (1.15-1.26), white meat RR } 0.87(0.65-1.17) \\
\text { and processed meat RR } 1.23(1.10-1.37) \text { and in women for red meat RR } 1.14(1.00-1.30) \text {, } \\
\text { white meat RR } 1.01(0.89-1.15) \text { and processed meat RR } 1.34(1.09-1.66) \text {. Risk of } \\
\text { cardiovascular mortality in men for red meat RR } 1.20(1.12-1.30) \text {, white meat RR } 1.05(0.74- \\
1.31) \text { and processed meat RR } 1.15(0.96-1.37) \text { and in women for red meat RR } 1.26(1.08- \\
1.47) \text {, white meat RR } 1.08(0.94-1.24) \text { and processed meat RR } 1.64(1.25-2.15) \text {. }\end{array}$ \\
\hline Xu $2018^{19}$ & Eggs & $\begin{array}{l}\text { The authors state "As no evidence suggested different associations by sex (P values for } \\
\text { interaction from } 0.45 \text { to } 0.92 \text { ), all analysis was conducted with both sexes combined, } \\
\text { adjusted for sex." }\end{array}$ \\
\hline Aune $2017^{20}$ & $\begin{array}{l}\text { Fruits and } \\
\text { vegetables }\end{array}$ & $\begin{array}{l}\text { Risk of CHD in men for fruits/vegetables RR } 0.93(0.89-0.97) \text {, fruits RR } 0.91(0.86-0.97) \text { and } \\
\text { vegetables RR } 0.77(0.68-0.89) \text { and in women for fruits/vegetables RR } 0.88(0.82-0.94) \text {, fruits } \\
\text { RR } 0.84(0.76-0.92) \text { and vegetables RR } 0.89(0.81-0.98) \text {. Risk of CVD in men for } \\
\text { fruits/vegetables RR } 0.93(0.85-1.03) \text {, fruits RR } 0.85(0.70-1.05) \text { and vegetables RR } 0.89(0.78- \\
1.00) \text { and in women for fruits/vegetables RR } 0.94(0.89-0.99) \text {, fruits RR } 0.83(0.77-0.90) \text { and } \\
\text { vegetables RR } 0.92(0.86-0.98) \text {. Risk of mortality in men for fruits/vegetables RR } 0.95 \text { (0.91- } \\
0.99) \text {, fruits RR } 0.88(0.78-1.00) \text { and vegetables RR } 0.91(0.84-0.99) \text { and in women for } \\
\text { fruits/vegetables RR } 0.94(0.90-0.98) \text {, fruits RR } 0.96(0.90-1.02) \text { and vegetables RR } 0.93(0.86- \\
0.99) \text {. }\end{array}$ \\
\hline $\begin{array}{l}\text { Schwingshackl } \\
2018^{21}\end{array}$ & Potatoes & Risk of CHD in men RR 1.05 (0.94-1.17) and women RR 1.00 (0.85-1.17). \\
\hline Ronksley $2011^{22}$ & Alcohol & $\begin{array}{l}\text { The authors state that sensitivity analyses confined to only studies of sex revealed generally } \\
\text { similar results for all the outcomes. }\end{array}$ \\
\hline Je $2014^{23}$ & Coffee & $\begin{array}{l}\text { For high vs low consumption, risk of mortality in men RR } 0.81(0.79-0.90) \text { and women RR } \\
0.84(0.79-0.89) \text {. }\end{array}$ \\
\hline Tang $2015^{24}$ & Tea & $\begin{array}{l}\text { For high vs low consumption, green tea and risk of CVD in men RR } 0.72(0.42-1.23) \text { and } \\
\text { women RR } 0.54(0.34-0.84) \text {. Green tea and risk of all-cause mortality in men RR } 0.80(0.68- \\
0.95) \text { and women RR } 0.74(0.60-0.93) \text {. Black tea and risk of CVD in men RR } 1.56(0.76-3.20) \\
\text { and women RR } 1.01(0.80-1.26) \text {. Black tea and risk of all-cause mortality in men RR } 1.45 \\
(0.95-1.21) \text { and women RR } 1.0(0.89-1.14) \text {. }\end{array}$ \\
\hline
\end{tabular}




\begin{tabular}{|l|l|l|}
\hline Narain $2016^{25}$ & Soft drink & Sex differences not explored for myocardial infarction or mortality. \\
\hline Guo $2017^{26}$ & Milk & No sex specific subgroup analyses were performed. \\
\hline Pimpin $2018^{7}$ & Butter & No sex specific subgroup analyses were performed. \\
\hline Aune $2016^{27}$ & Nuts & $\begin{array}{l}\text { Risk of CHD in men was RR 0.70 (0.62-0.80) and in women it was RR 0.71 (0.61-0.82). Risk of } \\
\text { CVD in men was RR 0.73 (0.66-0.81) and in women it was RR 0.86 (0.72-1.03). Risk of } \\
\text { mortality in men was RR 0.76 (0.70-0.83) and in women was RR 0.76 (0.64-0.88). }\end{array}$ \\
\hline Aburto 201328 & Salt & No sex specific subgroups reported for CVD and mortality. \\
\hline $\begin{array}{l}\text { Threapleton } \\
2013^{29}\end{array}$ & Fibre & $\begin{array}{l}\text { The authors state that for total fibre and CHD risk there was no differences observed } \\
\text { between the sexes. }\end{array}$ \\
\hline Malerba 201330 & Coffee & $\begin{array}{l}\text { Risk of mortality with incremental increase in coffee (1 cup/day), for men was RR 0.97 (0.95- } \\
0.99) \text { and for women it was RR 0.95 (0.93-0.97). Risk of CVD mortality, for men was RR 0.99 } \\
\text { (0.95-1.03) and for women it was RR 0.94 (0.92-0.98). }\end{array}$ \\
\hline $\begin{array}{l}\text { Martinez- } \\
\text { Gonzalez 201431 }\end{array}$ & Olive oil & $\begin{array}{l}\text { The authors state that no substantial differences were found for the risk of CVD when } \\
\text { separating the studies according to women or men. }\end{array}$ \\
\hline Yan 20178 & Soy & $\begin{array}{l}\text { Risk of CVD for soy intake in men was RR 0.91 (0.79-1.05) and for women it was RR 0.83 } \\
\text { (0.69-0.99). }\end{array}$ \\
\hline Ren 2018 32 & Chocolate & $\begin{array}{l}\text { Risk of CVD for chocolate consumption in men was RR 0.991 (0.964-1.019) and in women it } \\
\text { was RR 0.965 (0.931-1.001). }\end{array}$ \\
\hline
\end{tabular}

$\mathrm{RR}=$ relative risk, $\mathrm{CHD}=$ coronary heart disease, $\mathrm{CVD}=$ cardiovascular disease 